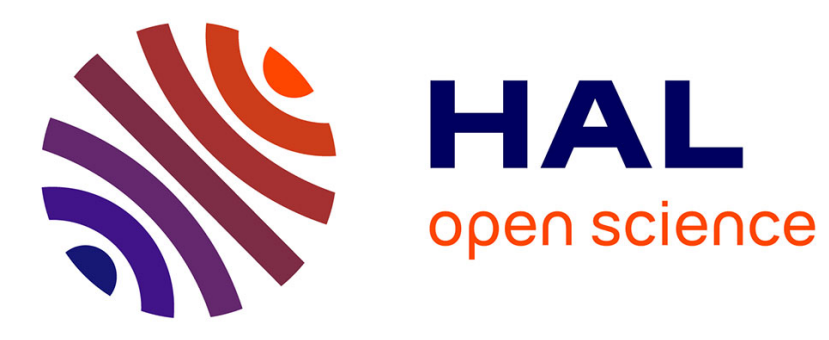

\title{
De la géochimie des productions provençales à pâte calcaire au référentiel régional
}

\author{
Anne Schmitt, Nadia Cantin, Valérie V. Thirion-Merle
}

\section{To cite this version:}

Anne Schmitt, Nadia Cantin, Valérie V. Thirion-Merle. De la géochimie des productions provençales à pâte calcaire au référentiel régional. Michel Pasqualini. Les céramiques communes d'Italie et de Narbonnaise Structures de production, typologies et contextes inédits IIè siècle av. J.C. - IIIè siècle ap. J.-C., 30, Diffusion De Boccard; l'ERMA di Bretschneider; M. D'Auria Editore; Edipuglia, 2009, Collection du Centre Jean Bérard, 978-2-903189-97-6. hal-01391494

\section{HAL Id: hal-01391494 https://hal.science/hal-01391494}

Submitted on 8 Nov 2016

HAL is a multi-disciplinary open access archive for the deposit and dissemination of scientific research documents, whether they are published or not. The documents may come from teaching and research institutions in France or abroad, or from public or private research centers.
L'archive ouverte pluridisciplinaire HAL, est destinée au dépôt et à la diffusion de documents scientifiques de niveau recherche, publiés ou non, émanant des établissements d'enseignement et de recherche français ou étrangers, des laboratoires publics ou privés. 


\title{
DE LA GEOCHIMIE DES PRODUCTIONS PROVENÇALES A PATE CALCAIRE AU REFERENTIEL REGIONAL
}

\author{
A. Schmitt, N. Cantin, V. Thirion-Merle \\ UMR 5138 "Archéométrie et Archéologie », CNRS, Université Lumière - Lyon 2, \\ Université de Lyon
}

\begin{abstract}
Résumé :
A partir de l'inventaire des ateliers connus en Provence ayant produits de la céramique commune, un échantillonnage a été réalisé pour définir les caractéristiques chimiques des principales productions à pâtes calcaires. Ainsi il a été démontré que les différents ateliers se distinguent nettement les uns des autres, du fait de l'emploi de matières premières spécifiques à leur production. L'étude a ainsi abouti à la constitution d'un référentiel régional des compositions géochimiques des céramiques provençales à pâte calcaire. Cette recherche constitue le préalable essentiel à une étude future de la diffusion de ces productions.
\end{abstract}

Les fouilles archéologiques menées en Provence ces dernières années ont mis en évidence que les céramiques communes à pâte calcaire provençales étaient issues de plusieurs ateliers et ce dès la deuxième moitié du $\mathrm{I}^{\mathrm{er}}$ S. av. J.-C..

Afin de mieux cerner les productions de chacun de ces centres, ainsi que les liens de commercialisation avec les autres sites consommateurs de la région, il est apparu nécessaire de caractériser plus précisément les ateliers.

Dans cette optique, au sein de l'Action Collective de Recherche sur les céramiques communes de Marseille à Gênes ( $\mathrm{II}^{\mathrm{e}}$ av. J.-C. - $\mathrm{III}^{\mathrm{e}}$ ap. J.-C.), un échantillonnage a été réalisé à partir d'exemplaires sélectionnés sur des critères typologiques parmi les ensembles de céramiques communes dites «à pâte claire » et les amphores. L'examen visuel de ces céramiques ne permettant pas à lui seul de distinguer des groupes, notamment en raison de la finesse des pâtes, des analyses physico-chimiques se sont avérées nécessaires.

L'objectif est de mettre en place un outil permettant ensuite de rechercher l'origine d'objets, typologiquement et chronologiquement comparables, découverts sur des sites de consommation. Nous nous sommes donc attachées à définir les critères de distinction entre les différents ateliers afin d'aboutir à la constitution d'un référentiel géochimique régional, préalable nécessaire aux questions de détermination d'origine. En outre, les analyses ont permis de discuter des ressources possibles en matières premières ainsi que de la notion de " pâtes claires ».

\section{Méthode d'analyse}

Lors de la table ronde de Naples, de nombreuses discussions ont porté sur la constitution de l'échantillonnage, la nature des pâtes, les méthodes d'analyse et les interprétations des résultats obtenus. Il est donc utile de préciser les raisons du choix de la méthode d'analyse employée ici.

L'examen visuel ou à la loupe binoculaire est une première étape nécessaire à l'étude des céramiques en laboratoire. Il permet d'obtenir un certain nombre d'informations sur les modes de façonnage et de cuisson des objets ainsi que sur la nature et les textures des pâtes. Cependant, ces seules observations ne sont pas suffisantes pour constituer des groupes 
représentatifs des centres de productions, surtout dans le cas de pâtes fines. C'est pourquoi on a le plus souvent recours à des méthodes d'analyses plus poussées.

Une première méthode d'analyse qui peut être mise en œuvre est l'analyse pétrographique qui fournit une identification des inclusions minérales contenues dans les pâtes des céramiques et permet ainsi de raisonner sur le contexte géologique des argiles utilisées. Cette méthode permet la caractérisation des productions et la constitution de groupes sur des critères pétrographiques. Elle permet, lors de comparaisons, de dire si les contextes géologiques sont compatibles. Mais elle est surtout efficace dans le cas de céramiques dont la pâte contient des inclusions grossières.

Dans le cas de céramiques à pâte fine, comme c'est le cas ici pour les céramiques communes provençales dites à pâte claire, l'analyse chimique est mieux adaptée. La méthode utilisée au laboratoire de l'UMR 5138 "Archéométrie et Archéologie" de Lyon est une méthode d'analyse chimique par fluorescence $X$ en dispersion de longueur d'onde'. Elle fournit la composition chimique globale de la céramique et par conséquent du matériau utilisé pour sa fabrication. L'analyse d'un seul individu est insuffisante pour caractériser très précisément une production. Il est nécessaire de constituer des échantillonnages importants sur les ateliers attestés afin d'étudier le rang de variation de chacun des constituants. Les résultats sont traités par des méthodes statistiques prenant en compte les dispersions par rapport à la moyenne. Il est alors possible de constituer des groupes de référence correspondant aux différents ateliers. La validité des traitements statistiques des données dépend du nombre de céramiques analysées (elle augmente avec le nombre de céramiques) et la qualité du résultat final est largement dépendant de la qualité de l'échantillonnage initial.

Les méthodes de statistique descriptive sont souvent suffisantes pour répondre à la question du nombre de groupes de pâte. L'étude de la dispersion des différents constituants chimiques en calculant des moyennes et des écarts par rapport à la moyenne (écart-type), et en visualisant la distribution des valeurs en construisant un histogramme est le premier pas nécessaire. On peut ensuite chercher les relations existantes entre les différents constituants en construisant des diagrammes binaires qui permettent de visualiser la nature des relations entre les groupes.

Mais, pour travailler sur plus de deux constituants chimiques à la fois, il est nécessaire d'utiliser des méthodes de traitement plus poussées, telles que les analyses statistiques multivariées couramment employées pour le traitement des données géochimiques (Swann et al., 1995). La méthode la plus classique est celle de la classification ascendante hiérarchique (ou cluster analysis) (Picon 1984, D'Anna et al. 2002 p.49 à 84). Elle repose sur la mesure de la ressemblance existant entre les différents exemplaires en tenant compte de tous les constituants chimiques et en leur donnant un poids équivalent et permet de mettre en évidence des groupes au sein desquels existent de fortes affinités de compositions. Les calculs sont effectués sur la matrice des données centrées réduites ce qui permet de donner le même poids à tous les constituants chimiques (qu'il s'agisse des constituants majeurs ou des éléments traces) et le calcul des différences repose sur la mesure de la distance euclidienne entre tous les exemplaires pris deux par deux. Lors des regroupements, on utilise les moyennes non pondérées. C'est ainsi que les deux premiers exemplaires les plus ressemblants sont regroupés pour former un " pseudo échantillon » dont la composition est celle de la moyenne des deux

\footnotetext{
${ }^{1} \mathrm{Au}$ laboratoire de l'UMR 5138 "Archéométrie et Archéologie" de Lyon, vingt-quatre éléments chimiques sont déterminés et les résultats de cette analyse chimique sont exprimés en pourcentages d'oxyde pour les constituants majeurs et en partie par million de métal pour les éléments traces. Il s'agit pour les éléments majeurs du calcium $(\mathrm{CaO})$, fer $\left(\mathrm{Fe}_{2} \mathrm{O}_{3}\right)$, titane $\left(\mathrm{TiO}_{2}\right)$, potassium $\left(\mathrm{K}_{2} \mathrm{O}\right)$, silicium $\left(\mathrm{SiO}_{2}\right)$, aluminium $\left(\mathrm{Al}_{2} \mathrm{O}_{3}\right)$, magnésium $(\mathrm{MgO})$, manganèse $(\mathrm{MnO})$, sodium $\left(\mathrm{Na}_{2} \mathrm{O}\right)$ et phosphore $\left(\mathrm{P}_{2} \mathrm{O}_{5}\right)$ et pour les éléments traces du zirconium $(\mathrm{Zr})$, strontium $(\mathrm{Sr})$, rubidium $(\mathrm{Rb})$, zinc $(\mathrm{Zn})$, chrome $(\mathrm{Cr})$, nickel $(\mathrm{Ni})$, lanthane $(\mathrm{La})$, baryum $(\mathrm{Ba})$, vanadium $(\mathrm{V})$, cérium $(\mathrm{Ce})$, yttrium $(\mathrm{Y})$, thorium $(\mathrm{Th})$, plomb $(\mathrm{Pb})$ et cuivre $(\mathrm{Cu})$.
} 
échantillons qui le forment, puis ce pseudo-échantillon est comparé à tous les autres exemplaires restants. Par itérations successives, les exemplaires les plus ressemblants se regroupent. Le résultat de cette classification est représenté sous forme d'un diagramme arborescent (dendrogramme) où chaque échantillon céramique est représenté par un trait vertical à la base du graphique. Il se rattache à son plus proche voisin à une hauteur proportionnelle à la distance mesurée entre leurs compositions chimiques. Sur le dendrogramme se détachent donc des groupes d'individus de compositions d'autant plus similaires qu'ils seront reliés à bas niveau. Tout échantillon rattaché à une hauteur relativement élevée à l'un ou l'autre de ces groupes aura une faible probabilité d'en faire partie.

A la suite de ces calculs, il est indispensable de compléter l'étude par un examen détaillé et approfondi des données individuelles de composition et de vérifier les raisons géochimiques des regroupements observés. Les groupes validés sont alors confrontés aux données archéologiques.

\section{2. Étude géochimique des ateliers provençaux}

\subsection{Echantillonnage}

L'étude des caractéristiques chimiques des ateliers gallo-romains de céramiques communes provençales à pâte claire a été réalisée à partir d'un échantillonnage des formes les plus typiques des céramiques communes mais également des amphores (Tab. 1). En effet, la vaisselle et les amphores peuvent avoir été produits dans les mêmes ateliers et parfois à partir du même matériau argileux.

Les sites d'ateliers échantillonnés sont d'ouest en est : Arles (Trinquetaille), Velaux (Moulin du Pont et plaine de Levrau), Marseille (Carmes), Aix-en-Provence (République et Palais Monclar), Fox-Amphoux, La Celle Revou, Lorgues, Bras, Fréjus (Sainte-Croix 2 et SaintLambert, Jardin d'Ariane) et Cannes (Villa Titi). Les sites de consommation étudiés pour comparaison sont Arles (Odéon), Marseille (Sainte-Barbe), Olbia et Nice (Cimiez) (fig. 1).

Des analyses réalisées antérieurement, au Laboratoire de Céramologie, ont été incluses à cet échantillonnage pour étoffer notre connaissance de la variabilité des compositions chimiques dans cette région (Marseille, Velaux, Fréjus, Cannes).

\subsection{Pâtes claires ?}

Soulignons tout d'abord qu'une observation macroscopique des pâtes a mis en évidence l'existence de lots de céramiques non calcaires, confirmés par l'analyse, à Fréjus, sur l'un des sites d'atelier (amphorettes du Jardin d'Ariane) et à Olbia (coupes Pasqualini 1a). Le classement de tous ces objets avec des céramiques calcaires dans une même catégorie typologique dite des «pâtes claires » ne nous semble donc pas appropriée.

Il paraît effectivement important d'apporter ici quelques précisions quant à l'expression "pâtes claires». En effet, celle-ci semble avoir été utilisée pour désigner une catégorie céramique sans prendre en compte l'aspect technique auquel elle fait référence. De fait, le terme de "pâte claire » est techniquement associé à des pâtes calcaires cuites en mode A (cuisson réductrice, post-cuisson oxydante). Il est généralement possible de distinguer les céramiques en pâte calcaire de celles en pâte non calcaire à partir de critères visuels de couleur. Les pâtes calcaires cuites en mode A ont une couleur qui varie du rouge clair ou orangé, au beige, au jaune paille, et même au verdâtre en fonction du degré de cuisson. Les pâtes non calcaires cuites dans les mêmes conditions ont une variation différente de rouge à rouge foncé, puis marron jusqu'au brun foncé (Picon 2002). Ces critères de couleur ne sont pas toujours suffisants pour statuer sur le caractère calcaire ou non de la pâte et il reste des cas où il n'est pas possible de se prononcer comme pour les objets peu cuits ou en pâte peu 
calcaire. Mais lorsqu'elle est possible, cette distinction entre pâte calcaire et pâte non calcaire est importante lors de la constitution des échantillonnages puisque les comparaisons et les attributions d'origine ne sont possibles qu'entre objets de même nature. Ainsi, dans la mesure où les ateliers étudiés ici ont tous, à l'exception du Jardin d'Ariane à Fréjus (amphorettes), utilisé uniquement des argiles calcaires pour leurs productions, aucune attribution n'a pu être faite pour les coupes Pasqualini la du site d'Olbia.

Il nous semblerait nécessaire de restreindre l'utilisation de l'expression "pâtes claires » aux céramiques calcaires cuites en mode A. Quant aux céramiques non calcaires, elles devront être classées dans des catégories techniques explicitement détaillées.

\subsection{Comparaisons des différents ateliers}

Après avoir analysé plusieurs références de chaque atelier et étudié les variations des compositions chimiques au sein de chaque groupe, il est important de s'intéresser à la nature géologique des terrains sur lesquels les différents ateliers sont implantés.

Afin d'évaluer les capacités de distinction entre les différents ateliers, nous avons recensé les grandes formations géologiques susceptibles de contenir des niveaux argileux exploitables par les potiers. Une étude exhaustive des argiles par échantillonnage sur le terrain est matériellement impossible à réaliser du fait de la multiplicité des sources d'argiles possibles (argiles marines, colluvions, alluvions) et de la variabilité géochimique selon les contextes de dépôt. Il est donc illusoire de songer à réaliser un référentiel exhaustif des argiles. Nous nous sommes donc appuyées sur les cartes géologiques au 1/50 $000^{\mathrm{e}}$ qui, si elles ne fournissent pas une cartographie précise des gisements argileux, permettent du moins de réfléchir en terme de zones de même âge géologique où les argiles peuvent s'être déposées dans les mêmes conditions et peuvent donc avoir des parentés géochimiques.

La cartographie simplifiée des principales formations géologiques dans lesquelles sont susceptibles de s'être formées les argiles permet de visualiser les aires de même âge géologique (fig. 1). Pour en faciliter la lecture, les formations superficielles et les alluvions n'ont pas été représentées. Parmi ces grands ensembles on distinguera le socle primaire et ses terrains cristallins métamorphiques et volcaniques (Massif des Maures et de l'Esterel notamment) qui peuvent fournir des matériaux argileux non calcaires quand ils sont altérés. Les terrains secondaires sont largement représentés sur la carte et comprennent plusieurs niveaux argileux. Des ressources en argiles calcaires existent notamment dans les marnes du Trias alors qu'au sein des calcaires jurassiques et crétacés, les niveaux marneux et argileux peuvent exister localement mais semblent moins abondants. Les terrains tertiaires (éocènes, oligocènes et miocènes) peuvent fournir des ressources en matières argileuses calcaires notamment dans la vallée de l'Arc (Velaux, Aix-en-Provence) et à Marseille. Enfin, les argiles marines pliocènes calcaires sont présentes dans plusieurs vallées côtières (vallée du Rhône, basse vallée de l'Argens, vallée de la Siagne, vallée du Var).

En confrontant ces données géologiques à la localisation des ateliers de potiers gallo-romains, il apparaît que certains ont un grand choix d'approvisionnement et que certaines officines, bien que géographiquement éloignées, peuvent avoir utilisé des argiles extraites des mêmes formations. Ainsi autour de Fréjus, trois types de ressources sont utilisables : les formations alluviales, les formations colluviales d'altération du massif cristallin et les argiles marines pliocènes qui tapissent la paléo-vallée de l'Argens. Ces dernières sont également présentes dans les vallées de la Siagne (Cannes) et du Var (Nice). La carte géologique indique que les ressources argileuses sont différentes en amont du bassin de l'Argens (Fox-Amphoux, Lorgues, Bras, La Celle Revou), où, dans un contexte secondaire, les niveaux argileux abondent mais également les niveaux d'altération et les alluvions. Enfin, plus à l'ouest, on notera que les argiles oligocènes affleurent tant dans la vallée de l'Arc (Velaux, Aix-en- 
Provence) qu'à Marseille. A Arles, les ressources en argiles existent à proximité, notamment dans les argiles marneuses pliocènes à l'ouest du delta du Rhône.

Toutes ces zones doivent donc être étudiées en détail pour vérifier s'il est possible de distinguer les différents ateliers, même dans les zones où la nature géologique des argiles est identique. C'est pourquoi nous présenterons les résultats par ensembles géologiques, en commençant par celui de Nice à Fréjus, puis celui de l'Argens et enfin celui de la vallée de l'Arc. 


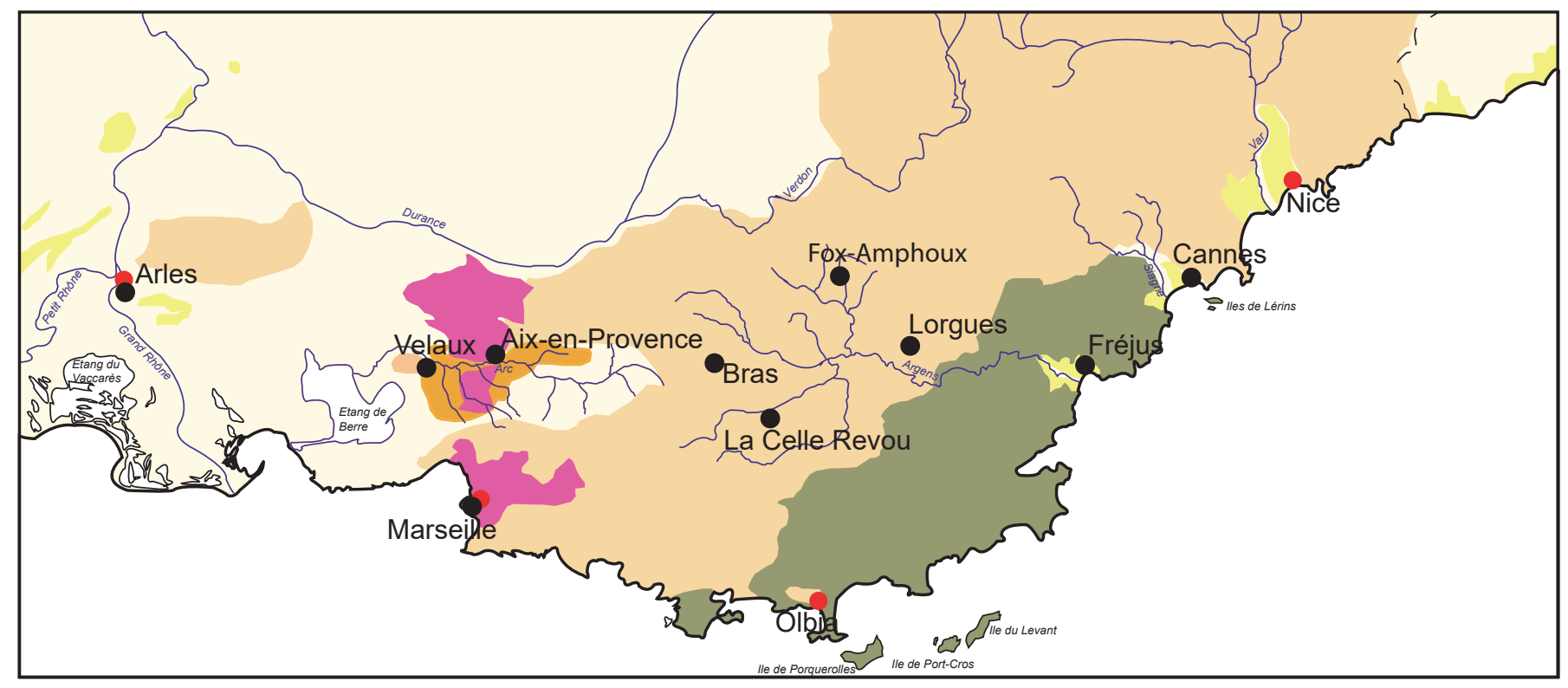

- Site d'atelier

- Site de consommation

Carte simplifiée des principales formations géologiques
Cristallin
Secondaire calcaire
\begin{tabular}{|l|l}
\hline Éocène \\
Oligocène \\
Pliocène
\end{tabular}
Miocène

Fig.1. 


\subsubsection{Aire de Nice-Cannes-Fréjus}

\section{Cas particulier du Jardin d'Ariane à Fréjus}

Comme nous l'avons déjà évoqué, un lot de céramiques non calcaires a été découvert sur le site du jardin d'Ariane à Fréjus considéré par les fouilleurs comme un atelier. Les quatre amphorettes analysées (ASE 32 à 35), ont une teneur moyenne en chaux $(\mathrm{CaO})$ de 1,48\%, alors que les références des autres ateliers de Fréjus sont calcaires, avec une teneur moyenne en chaux de 11,71\% (tous ateliers confondus) (Tab. 2). Deux amphores de type Gauloise 4 de ce site ont également été analysées. Elles se sont révélées calcaires et de composition comparable à celle des références des autres ateliers de Fréjus. Ainsi, au Jardin d'Ariane, coexistent deux types de production, l'une en pâte calcaire avec une argile similaire à celle utilisée dans les autres ateliers de la ville, l'autre en pâte non calcaire utilisant donc une autre argile. Cette distinction nette entre les deux types de pâte est d'ailleurs perceptible à l'œil nu (fig. 2). La présence d'argile non calcaire dans la région de Fréjus est attestée dans les formations d'altération des massifs cristallins et dans certaines alluvions mais il n'est pas possible de préciser la source exacte d'approvisionnement. Les amphorettes sont-elles des productions d'un atelier local utilisant ces ressources ou s'agit-il simplement d'objets importés? On peut également se poser la question de l'intérêt d'utiliser une argile non calcaire pour ces amphorettes. Pourquoi les potiers ont-ils fait ce choix : argile disponible à proximité immédiate de l'atelier, meilleur contrôle de la cuisson, choix esthétique, pratique locale?

\section{Comparaison entre Fréjus, Cannes et Nice}

Dans les vallées de l'Argens, de la Siagne et du Var existent des gisements d'argiles pliocènes partiellement masqués par les alluvions récentes. Ces argiles se sont déposées au fond des paléovallées côtières en milieu marin lors de la transgression pliocène. Ces gisements ont été utilisés à Fréjus (basse vallée de l'Argens) et probablement à Cannes (vallée de la Siagne). A Nice (vallée du Var), aucun atelier n'est archéologiquement connu, mais les marnes plaisanciennes sont connues pour avoir été exploitées pour la production céramique (Gèze et Nesteroff 1968). Le dépôt de ces argiles s'est fait à la même période géologique dans des milieux similaires, leurs compositions chimiques peuvent donc présenter des similitudes. Il convient donc de vérifier les possibilités de distinction entre les productions céramiques réalisées à partir des matériaux issus des niveaux pliocènes dans ces trois vallées.

La classification ascendante hiérarchique des exemplaires prélevés dans les ateliers de Cannes (Villa Titi, mais également site de Saint-Cassien) et de Fréjus (Saint-Lambert, Sainte-Croix, Porte d'Orée, Valescure) ${ }^{2}$ et dans un site d'habitat de Nice (Cimiez) met en évidence la bonne séparation de Cannes et Fréjus et la dispersion des exemplaires de Nice (fig. 3). Ici, les références de Fréjus, au sein desquelles il est difficile de réellement distinguer les différents ateliers, forment un grand groupe relativement homogène sur la gauche du graphique. Tous les ateliers ont probablement utilisé des argiles provenant des mêmes niveaux géologiques ${ }^{3}$. Cependant, quatre exemplaires diffèrent légèrement et se regroupent sur la droite de la grappe.

\footnotetext{
${ }^{2}$ Afin d'étoffer notre étude des variations de compositions chimiques à Cannes et Fréjus, des références existant déjà dans la base de données de l'UMR 5138 "Archéométrie et Archéologie" ont été comparées aux analyses réalisées dans le cadre de l'ACR (Picon 1991).

${ }^{3}$ Les travaux de M. Ricq de Bouard ont démontré à plusieurs reprises qu'il est très difficile de différencier les argiles de la région de Fréjus par l'analyse pétrographique ou chimique (Ricq de Bouard et al, 1993 et 1996). Seules les analyses des minéraux lourds semblent pour l'instant avoir apporté des indices dans la distinction des argiles employées ; notamment l'atelier de Valescure 1 se distinguerait de ceux de Saint-Lambert 3 et 7 (Ricq de Bouard et al., 1993 et 1996).
} 


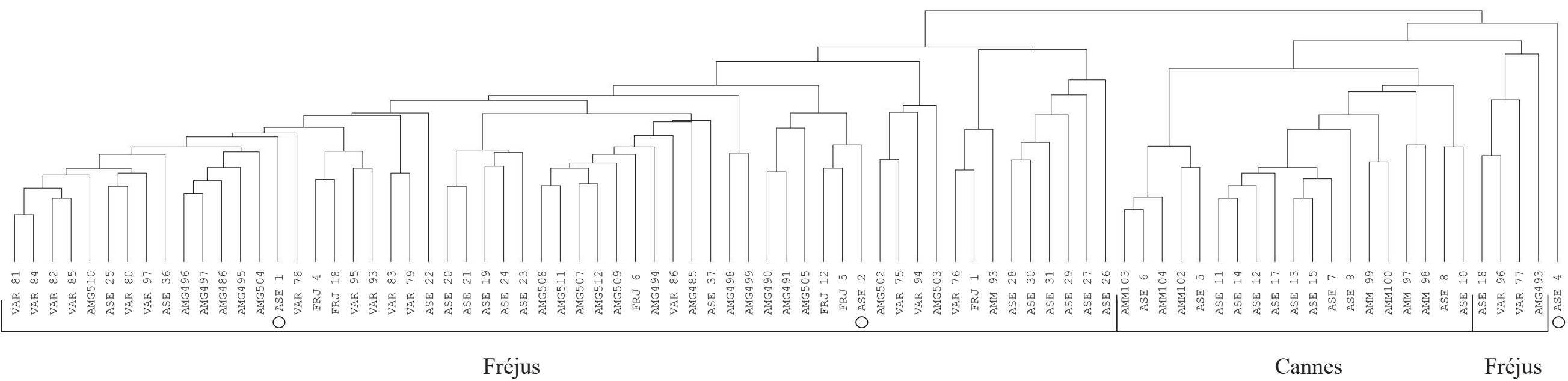

Fig.3 
L'ensemble des céramiques échantillonnées à Cannes n'est pas très homogène (Tab. 2) mais se distingue nettement des productions fréjusiennes. L'étude détaillée des caractéristiques chimiques a montré que les coupes Pasqualini 1a (Villa Titi-1), découvertes sur le site de la Villa Titi, ont des compositions qui ressemblent plus à celles des amphores du site de SaintCassien qu'à celles des amphores (Villa Titi-2) découvertes dans les fouilles de la Villa Titi. Y-a-t-il eu utilisation à la Villa Titi de deux types d'argile, l'un pour les coupes, l'autre pour les amphores, ou bien les coupes ont-elles été produites sur un autre site cannois, comme Saint-Cassien par exemple?

Deux des trois exemplaires prélevés sur le site de Cimiez à Nice (ASE 1 et 2, représentés par un rond blanc) se dispersent au sein du groupe de Fréjus, le troisième exemplaire (ASE 4) se plaçant en position complètement marginale sur la droite de la grappe (fig. 3).

Typologiquement les céramiques prélevées à Nice appartiennent aux mêmes types que les références de Fréjus, ce qui serait un argument pour l'attribution de ces exemplaires à Fréjus. Mais il pourrait également s'agir de productions niçoises qui ne se distingueraient pas de celles de Fréjus du fait de l'utilisation d'une argile de même nature géologique. L'attribution de ces individus à Fréjus nécessiterait de vérifier si les argiles pliocènes de Nice ont des compositions chimiques différentes de celles de Fréjus. En l'état actuel de nos maigres connaissances des compositions des argiles de Nice et surtout de l'échantillonnage très réduit, il est difficile de trancher entre ces deux hypothèses.

\subsubsection{Bassin de l'Argens}

Les céramiques à pâte calcaire du bassin de l'Argens (Fox-Amphoux, Bras, La Celle-Revou, Lorgues) présentent de fortes ressemblances typologiques avec les productions des ateliers de Fréjus (Pasqualini 1998). La classification d'après les compositions chimiques montre une séparation très nette entre le groupe de Fréjus, sur la gauche du graphique, et les ateliers situés en amont de l'Argens qui se placent dans un ensemble sur la droite (fig. 4). Les matières premières utilisées à Fox-Amphoux, Lorgues, Bras et La Celle Revou sont donc très différentes de celles de Fréjus (Tab. 2). Ceci est en accord avec ce que l'on sait des argiles pliocènes qui se sont déposées en milieu marin, au fond des paléovallées côtières. Elles sont très abondantes à Fréjus au débouché de l'Argens, mais ne remontent pas très haut dans la vallée. Dans le nord du bassin versant de l'Argens, les formations calcaires de l'ère secondaire contiennent différents niveaux argileux (marnes du Trias et du Jurassique). Les différences chimiques de ces dernières avec les marnes pliocènes sont importantes et portent essentiellement sur les taux d'oxydes de fer et de titane, d'alumine et de magnésie, nettement plus élevés pour les ateliers situés en amont du bassin de l'Argens.

Ainsi, le groupe céramique du bassin de l'Argens semble homogène d'un point de vue typologique mais il correspond à des productions d'ateliers différents qui utilisent des argiles calcaires locales distinctes. Les compositions chimiques des ateliers de l'Argens se distinguent entre elles et sont totalement différentes de celles des argiles pliocènes utilisées dans les ateliers de Fréjus.

\subsubsection{Comparaison des ateliers de la vallée de l'Arc et de Marseille}

Les argiles oligocènes du bassin de Marseille affleurent sur une aire géographique assez large jusqu'à Aix-en-Provence et ont été utilisées jusqu'à une période récente pour la fabrication de poteries. Pour assurer les possibilités de distinction entre ces deux régions, il faut donc vérifier si les ateliers de Marseille et ceux de la vallée de l'Arc (Velaux et Aix-en-Provence) utilisaient ou non le même type d'argile.

La comparaison des compositions chimiques montre la très bonne séparation entre les différents groupes (fig. 5). On remarque que les productions marseillaises (atelier de la Butte 


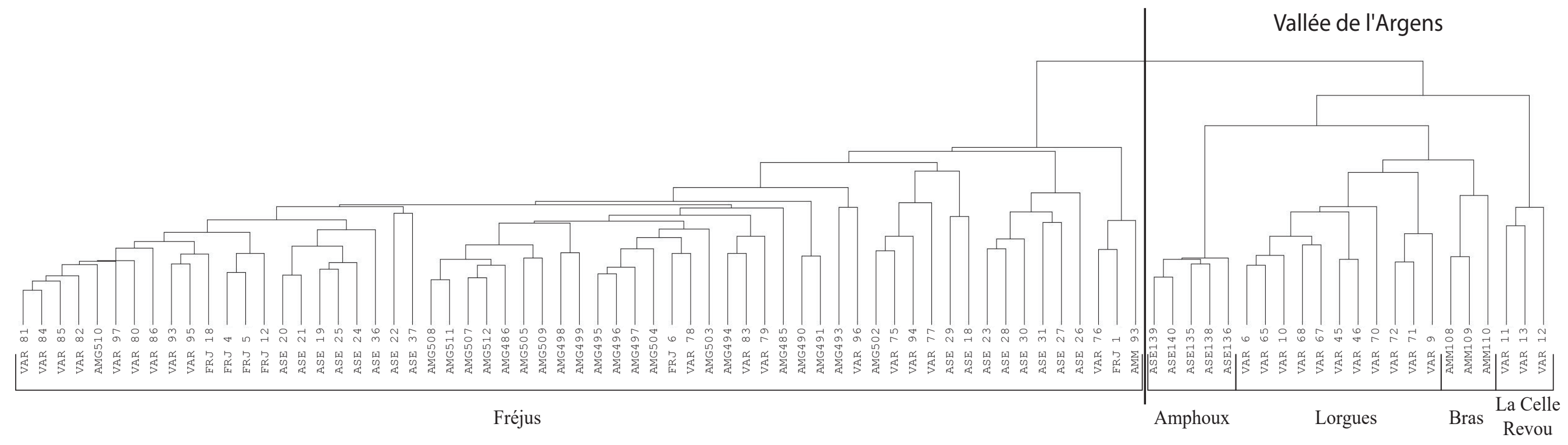

Fig.4 


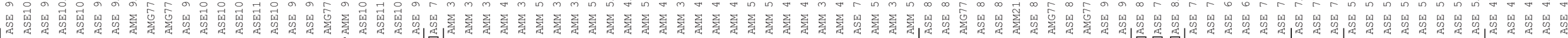

\section{Velaux}

Moulin du Pont

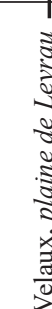

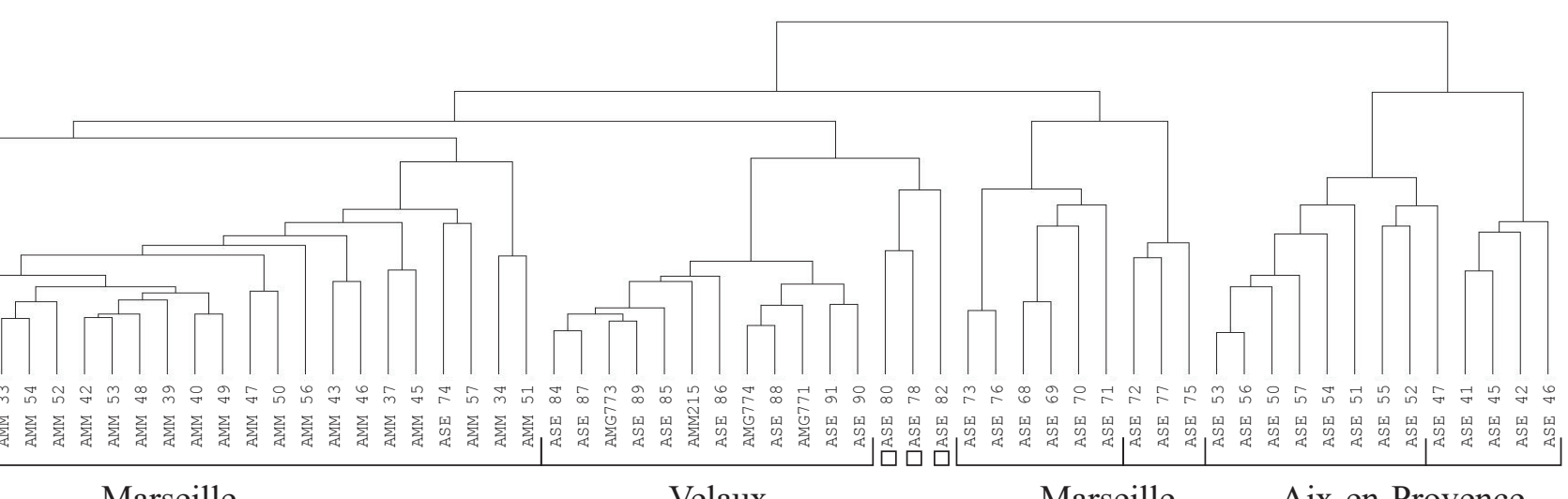

Marseille

Carmes-1

plaine de Levrau

Marseille

Aix-en-Provence

Carmes-3 Carmes-2 République Monclar 
des Carmes) se répartissent en trois groupes (Carmes-1, Carmes-2, Carmes-3) 4 $^{4}$ Cependant ces trois groupes restent très proches d'un point de vue géochimique, ce qui permet de soupçonner l'utilisation d'argiles issues des mêmes formations (Tab. 2). On note que l'amphore Bertucchi 6A (ASE 75) découverte sur la Butte des Carmes à Marseille a une composition chimique différente de celle des Bertucchi 6A des ateliers de Velaux (ASE 84 à ASE 86 ; ASE105) (Tab. 3). Par ailleurs, les céramiques découvertes à Sainte-Barbe en contexte de nécropole ne ressemblent manifestement pas aux productions de l'atelier de la Butte des Carmes. Elles pourraient cependant correspondre à une production régionale du fait de la variabilité de la composition des argiles oligocènes.

Les productions des deux ateliers de Velaux (plaine de Levrau et Moulin du Pont), se distinguent nettement (Tab. 2) comme le laissaient supposer les différences de texture et de couleur de pâte (fig. 6).

Sur la droite de la grappe de la figure 5, les ateliers d'Aix-en-Provence forment un ensemble bien individualisé (Nin et al. 2003). On peut néanmoins noter que les céramiques des ateliers de Monclar et de République se séparent nettement les unes des autres, les deux ateliers ne se sont pas approvisionnés sur le même gisement d'argiles (Tab. 2).

Les différences de composition chimique entre Marseille et la vallée de l'Arc sont importantes; les argiles utilisées sont donc différentes. A Marseille, l'étude des argiles prélevées localement a montré que ce sont bien les argiles oligocènes, aisément disponibles sur de fortes épaisseurs, qui ont été employées (Picon 1997). Celles-ci sont litées et contiennent des niveaux plus ou moins calcaires, ce qui explique la dispersion des compositions. Dans la vallée de l'Arc, on trouve des argiles calcaires dans les niveaux éocènes et dans les formations marno-calcaires du Secondaire. En l'absence de données précises sur la composition de ces matériaux, il est impossible de se prononcer plus précisément sur les lieux d'approvisionnement.

\subsubsection{Arles - l'atelier de Trinquetaille et le site de l'Odéon}

A Arles, les sept céramiques communes (coupes Pasqualini 1a) analysées de l'atelier de Trinquetaille forment un groupe de composition homogène (Tab. 2). Par contre, les trois céramiques découvertes sur le site de l'Odéon (cruches Pasqualini A1) ont des compositions dispersées très différentes (Tab. 3) et ne peuvent être attribuées à l'atelier de Trinquetaille.

\section{Référentiel géochimique régional}

Après avoir étudié en détail les compositions chimiques de chaque atelier et identifié les exemplaires marginaux (Tab. 3), nous avons réalisé une classification ascendante hiérarchique de l'ensemble des références (fig. 7). Cette classification a fait apparaître clairement un certain nombre de groupes, et a permis de se faire une bonne idée des possibilités de distinction entre les différents ateliers. En effet, les groupes de composition chimique identifiés dans la classification, correspondent aux productions des ateliers, à l'exception de Marseille dont les productions se répartissent dans trois groupes. Il a donc été démontré que les différents centres de production se séparent nettement et que les risques de confusion entre leurs produits sont minimes, sur le plan des compositions chimiques.

A partir de ces résultats, nous disposons d'un référentiel géochimique régional des céramiques communes et amphores à pâte calcaire provençales, défini par les caractéristiques de chaque atelier et ses différences par rapport à chacun des autres. Notons que ce référentiel est

\footnotetext{
4 Afin d'étoffer notre étude des variations de compositions chimiques dans les argiles de Marseille, des références existant déjà dans la base de données de l'UMR 5138 "Archéométrie et Archéologie" ont été comparées aux analyses réalisées dans le cadre de l'ACR.
} 
Figure 6

Fig.6a.

Fig.6b. 

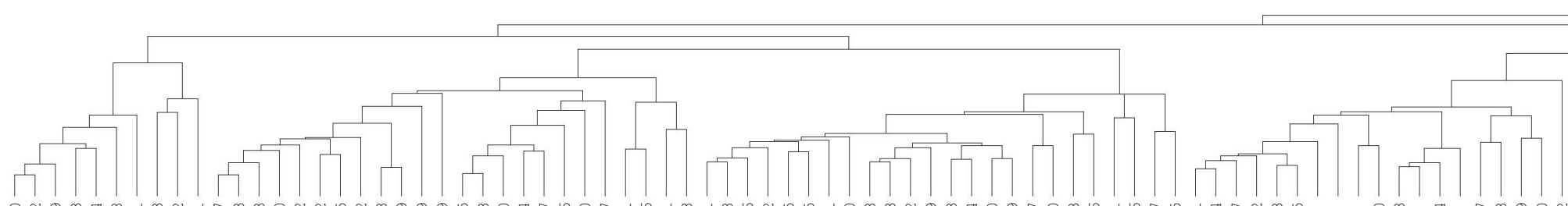

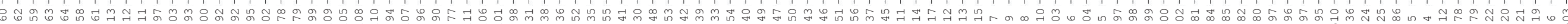

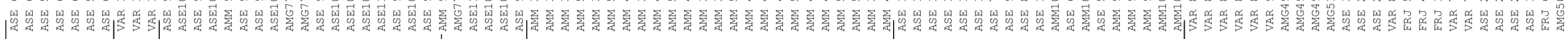

\section{Arles La Celle} Trinquetaille Revou

\begin{tabular}{|c|}
\hline $\begin{array}{c}\text { Velaux } \\
\text { Moulin du Pont }\end{array}$ \\
\hline
\end{tabular}

Marseille Carmes-1

Cannes

Fréjus
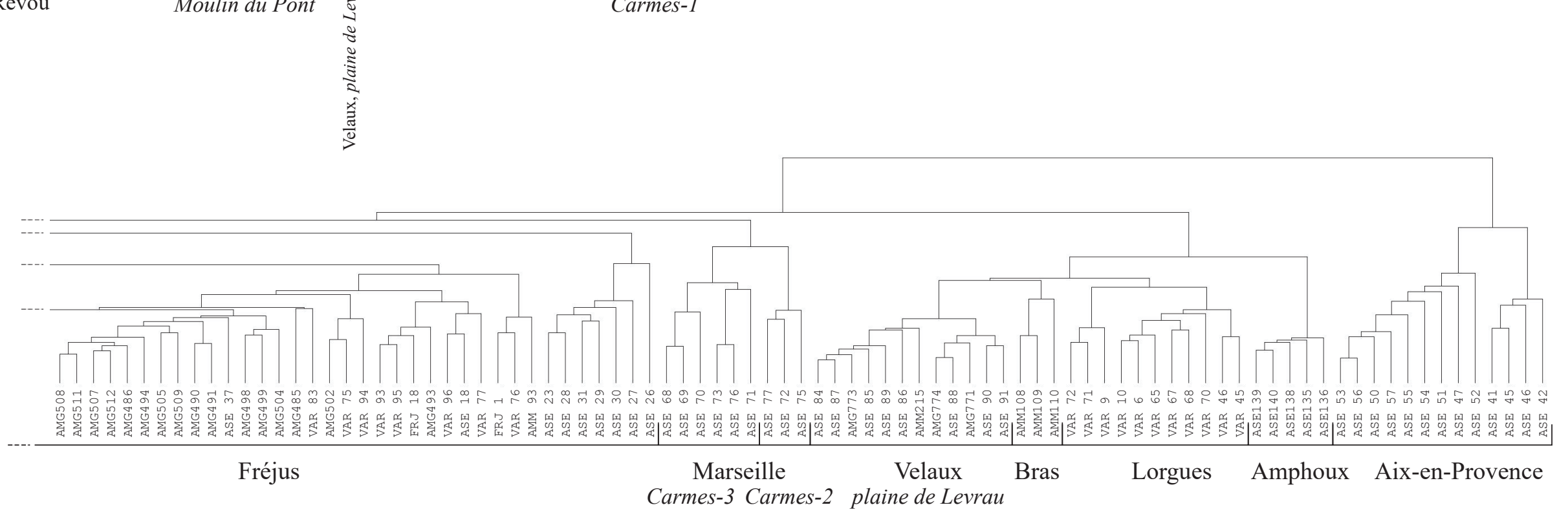
assurément partiel du fait que tous les ateliers producteurs de céramiques communes galloromaines provençales ne sont pas connus et encore moins analysés.

\section{Conclusion}

Pour utiliser un tel réseau de références à des fins d'attribution d'origine, notre étude a démontré qu'il était nécessaire d'effectuer des échantillonnages adaptés, en tenant compte à la fois des aspects macroscopiques des pâtes et de la typologie et en choisissant des exemplaires comparables à ceux connus sur les sites d'ateliers. En effet, une étude fine de la typologie conjointement avec celle des pâtes permet souvent de distinguer la variété des approvisionnements sur un site. Ainsi, les premiers essais de recherche d'origine de céramiques communes de Ligurie réalisés sans tenir compte de ces critères se sont révélés décevants. Parmi les exemplaires analysés découverts sur les sites de Vintimille, Albenga, Vado Ligure, Caprauna, San Bartolomeo, aucun n'a pu être attribué avec certitude aux ateliers référencés, à l'exception d'un tesson se rapprochant de Fréjus. Pour constituer l'échantillonnage des céramiques à analyser, il aurait fallu s'appuyer sur les groupes de pâtes déjà identifiés en Ligurie et décrits par analyse pétrographiques (Gandolfi et al. 2004).

Par ailleurs, il est établi à présent que l'appellation de " céramiques communes à pâte claire » ne convient pas pour désigner l'ensemble de ces productions. Il faudrait impérativement distinguer, dès l'étude céramologique, les objets réalisés en pâte calcaire et ceux réalisés en pâte non calcaire. Le terme de "pâte claire " ne pouvant pas regrouper les deux natures de pâtes, les céramiques échantillonnées devront donc être considérées comme des productions différentes.

Certaines questions restent encore en suspens et devront être développées. En premier lieu, il faudrait éclaircir les liens entre la situation géographique et la nature des sites dans lesquels sont implantés les ateliers et leurs possibilités de diffusion locale et/ou à longue distance.

De plus, il conviendrait de réfléchir à l'association des céramiques et des amphores. En effet, on connaît plusieurs ateliers produisant ces deux catégories de céramiques, mais on peut supposer que les voies de commercialisation ne sont pas les mêmes; les amphores étant essentiellement transportées pour leur contenu selon des circuits particuliers à la différence des céramiques communes principalement diffusées pour un usage domestique, mais parfois aussi pour leur contenu (D'Anna et al. 2003, p.147). Ainsi les travaux de F. Laubenheimer, associant données archéologiques et analyses, montrent que les amphores gauloises de Fréjus arrivent jusqu'au port d'Ostie et sont peu diffusées vers le Nord de la Gaule (Laubenheimer et Schmitt à paraître).

Qu'en est-il de la diffusion des céramiques communes provençales? Pour aborder cette question, une des voies pourrait être de se concentrer sur l'étude d'une forme précise, bien identifiée, et présente en abondance à la fois en Provence et dans les régions voisines. L'objectif serait de déterminer sa répartition sur chaque site, son abondance ainsi que les variétés d'aspect des pâtes. A partir de ces données et de la constitution d'un échantillonnage raisonné, la comparaison avec le référentiel géochimique provençal permettrait d'apporter des éléments de réponses à la question de la circulation des céramiques communes provençales. 


\section{Bibliographie}

D'Anna et al. 2003 : D'ANNA (A.), DESBAT (A.), GARCIA (D.), SCHMITT (A.), VERHARGHE (F.), La Céramique, la poterie du Néolithique aux temps modernes, éditions ERRANCE, collection "Archéologiques", 2003, 49-82, 147.

Gandolfi et al. 2004 : GANDOLFI (D.), GERVASINI (L.), CAPELLI (C.), La ceramica d'uso commune della stipe votiva di Caprauna (Alto Cuneo, Italia), una relettura dei dati archeologici e archeometrici. In : SFECAG, actes du congrès de Vallauris, 2004, 33-48.

Gèze et Nesteroff 1968 : GEZE (B.), NESTEROFF (W.), Carte géologique de Menton-Nice, XXXVII-42-43, 1:50000, 1968.

Laubenheimer et Schmitt à paraître : LAUBENHEIMER (F.), SCHMITT (A.), Amphores vinaires de Narbonnaise, production et grand commerce. Création d'une base de données géochimiques des ateliers, Travaux de la Maison de l'Orient et de la Méditerranée, à paraître.

Nin et al. 2003 : NIN (N.), PASQUALINI (M.), PESTY (M-T.), Les céramiques à pâte claire d'Aix-en-Provence et du bassin de l'Arc. Rebuts d'une officine de potier aux 38-42, boulevard de la République. In : Revue archéologique de Narbonnaise, 2003, 36, 283-304 .

Pasqualini 1998 : PASQUALINI (M.), en collaboration avec CARRE (M.-B.) et MASSON (L.), Les céramiques communes en basse Provence ( ${ }^{\mathrm{er}}-\mathrm{III}^{\mathrm{e}}$ siècles de notre ère), essai de classification. In : Fouilles à Marseille : contextes et mobiliers (I ${ }^{e r}$-VII ${ }^{e}$ siècles), M. Bonifay, M. B. Carre, Y. Rigoir (dir.), Etudes Massaliètes, 5, 1998, 293-308

Picon 1984 : PICON (M.), Le traitement des données d'analyse. In : Datation-caractérisation des céramiques anciennes. Cours postgradué européen. Bordeaux-Talence 1981. PACT 10. (dir. T. Hackens et M. Schvoerer) 1984, Paris, CNRS, 1984, 379-399.

Picon 1991 : PICON (M.), Analyses d'amphores de Fréjus. In : F. Laubenheimer, I. Béraud et C. Gébara coll., Les vides sanitaires et les amphores de la Porte d'Orée à Fréjus (Var), Gallia 48, 1991, 257-259.

Picon 1997 : PICON (M.), Analyses géochimiques. In : Marseille, les ateliers de potiers du $X I I I^{e}$ s. et le quartier Sainte-Barbe (V ${ }^{e}-X V I I^{e}$ s.), DAF 65, 1997, 170-173.

Picon 2002 : PICON (M.), Les modes de cuisson, les pâtes et les vernis de la Graufesenque : une mise au point. In: Céramiques de la Graufesenque et autres productions d'époque romaine. Nouvelles recherches. Hommage à Bettina Hoffmann (dir. M. Genin et A. Vernhet), ed . Monique Mergoil, 2002, 139-163.

Ricq de Bouard et al. 1993 : RICQ DE BOUARD (M.), BEINER (M.), DUBAR (M.), RICQ (J.-C.), ROSCIAN (S.), Argiles et ateliers gallo-romains dans la région de Fréjus et ses abords, Programme Fréjus-Argens, rapport, 1993, 22 p.

Ricq de Bouard et al. 1996 : RICQ DE BOUARD (M.), RICQ (J.-C.), BEINER (M.), Rapport d'analyses de céramiques de 6 sites de Fréjus, 1996.

Swann et al. 1995 : SWANN (A.R.H.), SANDILANDS (M..), Introduction to Geological Data Analysis, Blackwell Science Ltd, 1995, 446 p. 


\section{Légendes des figures et tableaux}

Fig. 1. Localisation des sites d'atelier et de consommation dans leur contexte géologique Fig. 2a. Pâte caractéristique des céramiques de Fréjus (ASE 29)

Fig. 2b. Pâte caractéristique des céramiques du site Jardin d'Ariane à Fréjus (ASE 34)

Fig. 3. Classification des céramiques communes et amphores des ateliers de Cannes et Fréjus et du site de consommation de Nice $(\mathrm{O})$

Classification ascendante hiérarchique en affinités moyennes non pondérées sur variables centrées réduites relatives aux 17 constituants chimiques suivants : $\mathrm{CaO}, \mathrm{Fe}_{2} \mathrm{O}_{3}, \mathrm{TiO}_{2}, \mathrm{~K}_{2} \mathrm{O}$, $\mathrm{SiO}_{2}, \mathrm{Al}_{2} \mathrm{O}_{3}, \mathrm{MgO}, \mathrm{MnO}, \mathrm{Zr}, \mathrm{Sr}, \mathrm{Rb}, \mathrm{Zn}, \mathrm{Cr}, \mathrm{Ni}, \mathrm{Ba}, \mathrm{V}$, Ce.

Fig. 4. Classification des céramiques communes de Fréjus et de la vallée de l'Argens (FoxAmphoux, Lorgues, Bras, La Celle Revou)

Classification ascendante hiérarchique en affinités moyennes non pondérées sur variables centrées réduites relatives aux 17 constituants chimiques suivants : $\mathrm{CaO}, \mathrm{Fe}_{2} \mathrm{O}_{3}, \mathrm{TiO}_{2}, \mathrm{~K}_{2} \mathrm{O}$, $\mathrm{SiO}_{2}, \mathrm{Al}_{2} \mathrm{O}_{3}, \mathrm{MgO}, \mathrm{MnO}, \mathrm{Zr}, \mathrm{Sr}, \mathrm{Rb}, \mathrm{Zn}, \mathrm{Cr}, \mathrm{Ni}, \mathrm{Ba}, \mathrm{V}, \mathrm{Ce}$.

Fig. 5. Classification des céramiques communes et amphores des ateliers de Velaux (Moulin du Pont et plaine de Levrau), Marseille (Butte des Carmes) et Aix-en-Provence (République et Monclar), ainsi que de la nécropole de Sainte-Barbe à Marseille ( $\square$ )

Classification ascendante hiérarchique en affinités moyennes non pondérées sur variables centrées réduites relatives aux 17 constituants chimiques suivants : $\mathrm{CaO}, \mathrm{Fe}_{2} \mathrm{O}_{3}, \mathrm{TiO}_{2}, \mathrm{~K}_{2} \mathrm{O}$, $\mathrm{SiO}_{2}, \mathrm{Al}_{2} \mathrm{O}_{3}, \mathrm{MgO}, \mathrm{MnO}, \mathrm{Zr}, \mathrm{Sr}, \mathrm{Rb}, \mathrm{Zn}, \mathrm{Cr}, \mathrm{Ni}, \mathrm{Ba}, \mathrm{V}, \mathrm{Ce}$.

Fig. 6a. Pâte caractéristique des céramiques du site plaine de Levrau à Velaux (ASE 86)

Fig. 6b. Pâte caractéristique des céramiques du site Moulin du Pont à Velaux (ASE 96)

Fig. 7. Classification de l'ensemble des céramiques communes et amphores des ateliers provençaux étudiés

Classification ascendante hiérarchique en affinités moyennes non pondérées sur variables centrées réduites relatives aux 17 constituants chimiques suivants : $\mathrm{CaO}, \mathrm{Fe}_{2} \mathrm{O}_{3}, \mathrm{TiO}_{2}, \mathrm{~K}_{2} \mathrm{O}$, $\mathrm{SiO}_{2}, \mathrm{Al}_{2} \mathrm{O}_{3}, \mathrm{MgO}, \mathrm{MnO}, \mathrm{Zr}, \mathrm{Sr}, \mathrm{Rb}, \mathrm{Zn}, \mathrm{Cr}, \mathrm{Ni}, \mathrm{Ba}, \mathrm{V}, \mathrm{Ce}$.

Tab. 1. Echantillonnage pris en compte dans l'étude (ASE = sigle correspondant aux échantillons analysés dans le cadre de l'ACR, autres sigles correspondant aux références antérieurement analysées).

Tab. 2. Compositions moyennes des ateliers ou groupes $\mathrm{m}=$ moyenne $; \sigma=$ écart-type $; \sigma \%=$ écart-type réduit $;$ na $=$ non analysé $; \mathrm{nc}=$ non calculé Tab. 3. Tableau des compositions chimiques de l'ensemble des céramiques étudiées et utilisées dans cette étude (sites de production et de consommation)

na $=$ non analysé

ASE* = exemplaire "marginal" d'un point de vue de la composition chimique, non pris en compte dans les calculs des moyennes du tableau 2 


\begin{tabular}{|c|c|c|c|}
\hline Ville & Site de découverte & $\mathrm{N}^{\circ}$ d'analyse & Description \\
\hline \multirow{8}{*}{ 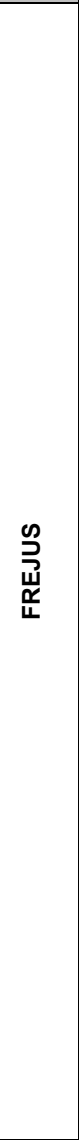 } & Sainte-Croix 2 & \begin{tabular}{|l|} 
ASE 18 \\
ASE 19 \\
ASE 20 \\
ASE 21 \\
ASE 22 \\
ASE 23 \\
ASE 24 \\
ASE 25 \\
\end{tabular} & $\begin{array}{l}\text { Amphore, Lenzbourg } \\
\text { Céramique commune, Pasqualini B1c } \\
\text { Céramique commune, Pasqualini 6a } \\
\text { Amphore, G8 } \\
\text { Amphore, G5 } \\
\text { Céramique commune, Pasqualini A1 } \\
\text { Amphore, G4 } \\
\text { Céramique commune, Pasqualini } 9 \\
\end{array}$ \\
\hline & Sainte-Croix & VAR 93 à VAR 97 & Amphore, G4 \\
\hline & \multirow[b]{2}{*}{ Saint-Lambert } & $\begin{array}{l}\text { ASE 26 } \\
\text { ASE 27 } \\
\text { ASE 28 } \\
\text { ASE 29 } \\
\text { ASE 30 } \\
\text { ASE } 31\end{array}$ & $\begin{array}{l}\text { Céramique commune, Pasqualini A1 } \\
\text { Céramique commune, Pasqualini B1 } \\
\text { Céramique commune, Pasqualini } 9 \\
\text { Amphore, G5 } \\
\text { Céramique commune, Pasqualini } 4 \\
\text { Amphore, G8 }\end{array}$ \\
\hline & & $\begin{array}{l}\text { VAR } 81 \text { à VAR } 86 \\
\text { AMG494 } \\
\text { AMG495 } \\
\text { AMG496 } \\
\text { AMG497 } \\
\text { AMG498 à AMG499 } \\
\text { AMG502 } \\
\text { AMG503 } \\
\text { AMG504 } \\
\text { AMG505 } \\
\text { AMG507 } \\
\text { AMG508 } \\
\text { AMG509 } \\
\text { AMG510 } \\
\text { AMG511 } \\
\text { AMG512 }\end{array}$ & $\begin{array}{l}\text { Amphore } \\
\text { Céramique commune, plaque Campana } \\
\text { Céramique commune, cruche à bec pincé } \\
\text { Céramique commune, vase à col évasé } \\
\text { Céramique commune, mortier } \\
\text { Amphore, Dr.2/4 } \\
\text { Amphore, Oberaden } 74 \\
\text { TUILE } \\
\text { Céramique commune, cruche à bec renflé } \\
\text { ARGILE } \\
\text { Céramique commune, coupe } \\
\text { Amphore, G5 } \\
\text { Céramique commune, cruche } \\
\text { Céramique commune } \\
\text { Céramique commune, jarre à fruits } \\
\text { Céramique commune, vase fermé à col évasé }\end{array}$ \\
\hline & Jardin d'Ariane & $\begin{array}{l}\text { ASE } 32 \text { à ASE } 35 \\
\text { ASE } 36 \text { à ASE } 37\end{array}$ & \begin{tabular}{|l|} 
Amphorette \\
Amphore, G4 \\
\end{tabular} \\
\hline & Pauvadou & $\begin{array}{l}\text { AMM } 93 ; \text { FRJ } 1 ; \\
\text { VAR } 75 \text { à VAR } 80\end{array}$ & Amphore, G5 \\
\hline & Porte d'Orée & \begin{tabular}{|l|} 
FRJ 4 \\
FRJ 5 \\
FRJ 6 \\
FRJ 12 \\
FRJ 18 \\
\end{tabular} & $\begin{array}{l}\text { Amphore, Dr.14 marque MARI } \\
\text { Amphore, Dr. } 2 / 4 \text { marque MARI } \\
\text { TUILE, marque MARI } \\
\text { Amphore } \\
\text { Amphore, Dr. } 2 / 4\end{array}$ \\
\hline & Valescure & $\begin{array}{l}\text { AMG485 à AMG486; } \\
\text { AMG490 } \\
\text { AMG491 } \\
\text { AMG493 }\end{array}$ & $\begin{array}{l}\text { Céramique commune, cruche à } 2 \text { anses } \\
\text { TUILE surcuite } \\
\text { ARGILE }\end{array}$ \\
\hline \multirow{4}{*}{ 怘 } & La Celle Revou & VAR 11 à VAR 13 & Amphore \\
\hline & Bras & AMM108 à AMM110 & Céramique commune, p.claire \\
\hline & Lorgues & $\begin{array}{l}\text { VAR } 6 \text { à VAR } 10 \\
\text { VAR } 45 \text { à VAR } 46 \\
\text { VAR } 63 \text { à VAR } 72 \\
\end{array}$ & \begin{tabular}{|l|} 
Amphore \\
indéterminé \\
Amphore
\end{tabular} \\
\hline & Fox-Amphoux & ASE135 à ASE140 & Céramique commune \\
\hline \multirow{3}{*}{ 岱 } & \multirow[t]{2}{*}{ Villa Titi } & ASE 5 à ASE 7 & Amphore, G6 \\
\hline & & ASE 8 à ASE 17 & Céramique commune, Coupe Pasqualini 1a \\
\hline & Saint-Cassien & $\begin{array}{l}\text { AMM } 97 \text { à AMM100 } \\
\text { AMM101 à AMM104 }\end{array}$ & $\begin{array}{l}\text { Amphore, Dr16 } \\
\text { Amphore, G6 }\end{array}$ \\
\hline \multirow{3}{*}{ 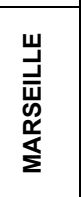 } & Carmes & $\begin{array}{l}\text { ASE } 68 \text { à ASE } 71 \\
\text { ASE } 72 \text { à ASE } 74 \\
\text { ASE } 75 \\
\text { ASE } 76 \text { à ASE } 77\end{array}$ & $\begin{array}{l}\text { Céramique commune, cruche Pasqualini A1 } \\
\text { Amphore, Bertucchi 7A } \\
\text { Amphore, Bertucchi 6A } \\
\text { Amphore, Bertucchi 7A }\end{array}$ \\
\hline & & $\begin{array}{l}\text { AMM } 30 \text { à AMM } 43 \\
\text { AMM } 45 \text { à AMM } 57\end{array}$ & $\begin{array}{l}\text { Céramique commune } \\
\text { Amphore }\end{array}$ \\
\hline & Sainte-Barbe & ASE 78 à ASE 82 & Céramique commune, coupe Pasqualini 1a \\
\hline \multirow{2}{*}{ 旡 } & Trinquetaille & ASE 58 à ASE 64 & Céramique commune, coupe Pasqualini 1a \\
\hline & Odéon & ASE 65 à ASE 67 & Céramique commune, cruche Pasqualini A1 \\
\hline \multirow[b]{2}{*}{$\frac{x}{<}$} & Monclar & ASE 41 à ASE 46 & indéterminé \\
\hline & République & $\begin{array}{l}\text { ASE } 47 \text { à ASE } 51 \\
\text { ASE } 52 \\
\text { ASE } 53 \text { à ASE } 57\end{array}$ & $\begin{array}{l}\text { indéterminé } \\
\text { Céramique commune, Pasqualini 6a } \\
\text { Amphore, Bertucchi 6B }\end{array}$ \\
\hline \multirow[b]{2}{*}{ 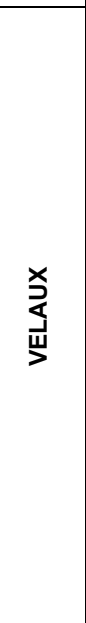 } & plaine de Levrau & $\begin{array}{l}\text { ASE } 84 \text { à ASE } 86 \\
\text { ASE } 87 \\
\text { ASE } 88 \text { à ASE } 90 \\
\text { ASE } 91 \\
\text { AMM214 à AMM215 } \\
\text { AMG769 à AMG770 } \\
\text { AMG771 } \\
\text { AMG772 à AMG775 }\end{array}$ & $\begin{array}{l}\text { Amphore, Bertucchi 6A } \\
\text { Amphore, Bertucchi 7A } \\
\text { Amphore, Bertucchi (imitation Dr. 7-11) } \\
\text { Amphore, Bertucchi (imitation Dr. 2/4) } \\
\text { Amphore } \\
\text { Amphore, Dr.2/4 } \\
\text { Amphore, G7 } \\
\text { Amphore, DR.7/11 }\end{array}$ \\
\hline & Moulin du pont & $\begin{array}{l}\text { ASE } 92 \text { à ASE } 94 \\
\text { ASE } 95 \text { à ASE } 97 \\
\text { ASE } 98 \\
\text { ASE } 99 \\
\text { ASE100 } \\
\text { ASE101 } \\
\text { ASE102 à ASE103 } \\
\text { ASE104 } \\
\text { ASE105 } \\
\text { ASE106 } \\
\text { ASE107 } \\
\text { ASE108 à ASE109 } \\
\text { ASE110 à ASE111 } \\
\text { AMM } 90 \text { à AMM } 92 \\
\text { AMG776 à AMG779 }\end{array}$ & $\begin{array}{l}\text { Amphore, Bertucchi 6B } \\
\text { Amphore, Bertucchi (imitation Dr. 2/4) } \\
\text { Amphore, Bertucchi 7A } \\
\text { Amphore, G2 } \\
\text { Amphore, G8 } \\
\text { Amphore, G4 } \\
\text { Amphore, Bertucchi (imitation Dr. 2/4) } \\
\text { Amphore, Bertucchi (imitation Dr. 2/4), marque } \\
\text { SENECA sur le col } \\
\text { Amphorette, Bertucchi 6A } \\
\text { Céramique commune, Pasqualini } 9 \\
\text { Céramique commune, Pasqualini 6A } \\
\text { Couvercle d'amphore } \\
\text { éléments de four } \\
\text { Amphore, Dr.2/4 } \\
\text { Amphore, G7 }\end{array}$ \\
\hline NICE & Cimiez, thermes & ASE 1 à ASE 4 & Céramique commune, cruches Pasqualini A1 \\
\hline YERES & Olbia & ASE 38 à ASE 40 & Céramique commune, coupe Pasqualini 1a \\
\hline
\end{tabular}




\begin{tabular}{|c|c|c|c|c|c|c|c|c|c|c|c|c|c|c|c|c|c|c|c|c|c|c|c|c|}
\hline \multicolumn{25}{|l|}{ Cannes } \\
\hline Nom & $\mathrm{CaO}$ & $\mathrm{Fe}_{2} \mathrm{O}_{3}$ & $\mathrm{TiO}_{2}$ & $\mathrm{~K}_{2} \mathrm{O}$ & $\mathrm{SiO}_{2}$ & $\mathrm{Al}_{2} \mathrm{O}_{3}$ & $\mathrm{MgO}$ & $\mathrm{MnO}$ & $\mathrm{Na}_{2} \mathrm{O}$ & $\mathrm{P}_{2} \mathrm{O}_{5}$ & $\mathrm{Zr}$ & $\mathrm{Sr}$ & $\mathrm{Rb}$ & $\mathrm{Zn}$ & $\mathrm{Cr}$ & $\mathrm{Ni}$ & La & $\mathrm{Ba}$ & V & $\mathrm{Ce}$ & $\mathrm{Y}$ & Th & $\mathrm{Pb}$ & $\mathrm{Cu}$ \\
\hline \multicolumn{25}{|c|}{ Villa Titi - $1(n=11)$} \\
\hline ASE 7 & 13.87 & 4.58 & 0.697 & 3.09 & 63.38 & 11.73 & 1.91 & 0.0346 & 0.42 & 0.12 & 314 & 303 & 155 & 44 & 64 & 31 & 40 & 474 & 58 & 85 & 37 & 4 & 72 & 14 \\
\hline ASE 8 & 15.60 & 4.00 & 0.606 & 2.52 & 64.20 & 10.44 & 1.89 & 0.0401 & 0.35 & 0.17 & 313 & 548 & 108 & 54 & 55 & 23 & 35 & 435 & 53 & 82 & 37 & 0 & 115 & 36 \\
\hline ASE 9 & 16.64 & 3.97 & 0.607 & 2.77 & 63.54 & 10.10 & 1.58 & 0.0260 & 0.42 & 0.15 & 317 & 331 & 107 & 45 & 53 & 21 & 42 & 352 & 61 & 98 & 39 & 0 & 564 & 21 \\
\hline ASE 10 & 17.17 & 3.91 & 0.595 & 2.73 & 63.07 & 10.22 & 1.59 & 0.0367 & 0.36 & 0.16 & 307 & 427 & 112 & 55 & 50 & 21 & 33 & 333 & 68 & 77 & 34 & 0 & 87 & 24 \\
\hline ASE 11 & 10.52 & 4.20 & 0.631 & 2.75 & 67.5 & 11.80 & 1. & 0 & 0. & 0.11 & 316 & 328 & 131 & 50 & 62 & 28 & 40 & 384 & 0 & 91 & 35 & 8 & 47 & 37 \\
\hline SE 12 & 12.77 & 4.45 & 0.664 & 2.78 & 4.9 & 11.83 & 1.80 & 28 & 0.4 & 0.11 & 330 & 324 & 137 & 57 & 66 & 27 & 41 & 340 & 72 & 85 & 37 & 0 & 95 & 20 \\
\hline ASE 13 & 12.95 & 4.43 & 0.663 & 3.03 & 64.61 & 11.72 & 1.79 & 0.0326 & 0.48 & 0.14 & 333 & 324 & 126 & 46 & 60 & 29 & 44 & 398 & 50 & 88 & 42 & 10 & 38 & 23 \\
\hline ASE 14 & 11.02 & 4.40 & 0.655 & 2.81 & 66.61 & 11.80 & 1.95 & 0.0389 & 0.45 & 0.11 & 317 & 349 & 135 & 48 & 56 & 27 & 37 & 342 & 62 & 89 & 37 & 6 & 70 & 21 \\
\hline ASE 15 & 13.81 & 4.26 & 0.651 & 2.71 & 64.83 & 11.26 & 1.71 & 0.0324 & 0.46 & 0.11 & 323 & 343 & 133 & 45 & 62 & 26 & 33 & 329 & 59 & 87 & 35 & 6 & 57 & 29 \\
\hline ASE $16^{*}$ & 14.42 & 4.50 & 0.646 & 3.34 & 61.89 & 11.88 & 2.36 & 0.1369 & 0.47 & 0.19 & 301 & 454 & 127 & 62 & 53 & 30 & 37 & 472 & 48 & 87 & 39 & 13 & 23 & 22 \\
\hline ASE 17 & 11.69 & 4.23 & 0.629 & 2.82 & 66.14 & 11.72 & 1.96 & 0.0439 & 0.50 & 0.11 & 308 & 224 & 132 & 53 & 57 & 27 & 39 & 328 & 63 & 87 & 34 & 8 & 67 & 18 \\
\hline \multicolumn{25}{|c|}{ Villa Titi - $2(n=5)$} \\
\hline AMM102 & 18.61 & 4.41 & 0.610 & 2.95 & 58.25 & 11.65 & 2.58 & 0.1065 & 0.49 & 0.17 & 287 & 408 & 127 & 44 & 58 & 35 & 20 & 400 & 66 & & na & na & na & na \\
\hline AMM103 & 18.14 & 4.47 & 0.640 & 2.94 & 58.04 & 12.39 & 2.50 & 0.0471 & 0.48 & 0.16 & 285 & 382 & 144 & 56 & 66 & 33 & 0 & 368 & 76 & $82 r$ & na & na & na & na \\
\hline AMM104 & 17.18 & 4.65 & 0.680 & 2.94 & 58.59 & 12.74 & 2.28 & 0.0425 & 0.59 & 0.12 & 313 & 362 & 149 & 51 & 66 & 34 & 0 & 325 & 82 & $84 r$ & na & na & na & na \\
\hline ASE 5 & 19.15 & 4.50 & 0.628 & 2.83 & 57.98 & 11.69 & 2.43 & 0.0458 & 0.40 & 0.18 & 279 & 373 & 137 & 48 & 57 & 28 & 40 & 433 & 66 & 82 & 39 & 3 & 64 & 17 \\
\hline ASE 6 & 17.80 & 4.70 & 0.664 & 2.90 & 58.13 & 12.41 & 2.59 & 0.0485 & 0.42 & 0.16 & 300 & 392 & 147 & 58 & 67 & 27 & 35 & 365 & 79 & 88 & 39 & 13 & 35 & 20 \\
\hline \multicolumn{25}{|c|}{ Saint-Cassien $(n=4)$} \\
\hline AMM 97 & 13.03 & 90 & 0.590 & 2.6 & & 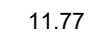 & & & & 19 & 269 & 284 & 95 & 60 & 38 & 31 & 42 & 431 & 61 & $72 r$ & na & na & na & na \\
\hline AMM 98 & & 3.92 & 0.580 & 2.8 & 68.02 & 12.08 & & & & 0.20 & 290 & 234 & 111 & 53 & 46 & 34 & 0 & 365 & 66 & & na & na & na & na \\
\hline AMM 99 & 14.37 & 4.21 & 0.630 & 3.10 & 62.76 & 12.29 & 1.61 & 0.0333 & 0.66 & 0.15 & 305 & 325 & 133 & 54 & 59 & 42 & 30 & 316 & 76 & $67 r$ & na & na & na & na \\
\hline AMM100 & 11.57 & 4.38 & 0.670 & 3.28 & 64.56 & 12.59 & 1.57 & 0.0292 & 0.79 & 0.35 & 316 & 287 & 141 & 60 & 58 & 28 & 30 & 442 & 75 & $75 r$ & na & na & na & na \\
\hline
\end{tabular}

\begin{tabular}{|c|c|c|c|c|c|c|c|c|c|c|c|c|c|c|c|c|c|c|c|c|c|c|c|c|}
\hline \multicolumn{25}{|l|}{ Fréjus } \\
\hline Nom & $\mathrm{CaO}$ & $\mathrm{Fe}_{2} \mathrm{O}_{3}$ & $\mathrm{TiO}_{2}$ & $\mathrm{~K}_{2} \mathrm{O}$ & $\mathrm{SiO}_{2}$ & $\mathrm{Al}_{2} \mathrm{O}_{3}$ & $\mathrm{MgO}$ & $\mathrm{MnO}$ & $\mathrm{Na}_{2} \mathrm{O}$ & $\mathrm{P}_{2} \mathrm{O}_{5}$ & $\mathrm{Zr}$ & $\mathrm{Sr}$ & $\mathrm{Rb}$ & $\mathrm{Zn}$ & $\mathrm{Cr}$ & $\mathrm{Ni}$ & La & $\mathrm{Ba}$ & V & $\mathrm{Ce}$ & $\mathrm{Y}$ & Th & $\mathrm{Pb}$ & $\mathrm{Cu}$ \\
\hline \multicolumn{25}{|c|}{ Tous ateliers confondus $(n=61)$} \\
\hline AMG485 & 14.09 & 4.92 & 0.682 & 3.19 & 58.23 & 16.37 & 1.35 & 0.0379 & 0.77 & 0.17 & 265 & 268 & 157 & 81 & 57 & 49 & 35 & 837 & 67 & & na & na & na & na \\
\hline AMG486 & 9.51 & 4.98 & 0.700 & 3.43 & 61.55 & 16.70 & 1.86 & 0.0512 & 0.91 & 0.15 & 278 & 267 & 157 & 82 & 54 & 54 & 42 & 421 & 72 & & na & na & na & na \\
\hline AMG490 & 12.51 & 5.12 & 0.689 & 3.00 & 58.66 & 16.85 & 1.75 & 0.0334 & 1.12 & 0.12 & 252 & 253 & 148 & 89 & 68 & 40 & 51 & 417 & 84 & $99 \mathrm{r}$ & na & na & na & na \\
\hline AMG491 & 15.58 & 4.91 & 0.667 & 2.88 & 56.16 & 16.44 & 1.90 & 0.0308 & 1.18 & 0.10 & 234 & 281 & 145 & 89 & 62 & 46 & 27 & 323 & 89 & & na & na & na & na \\
\hline AMG493 & 12.05 & 4.27 & 0.544 & 3.09 & 63.76 & 13.62 & 1.39 & 0.0364 & 1.04 & 0.07 & 250 & 184 & 168 & 82 & 51 & 43 & 26 & 342 & 76 & $67 r$ & na & na & na & na \\
\hline AMG494 & 8.14 & 5.38 & 0.761 & 3.54 & 61.94 & 17.48 & 1.64 & 0.0364 & 0.84 & 0.09 & 273 & 273 & 173 & 96 & 55 & 55 & 52 & 509 & 76 & $88 r$ & na & na & na & na \\
\hline AMG495 & 10.37 & 4.82 & 0.672 & 3.25 & 61.65 & 16.16 & 1.74 & 0.0602 & 0.94 & & 248 & 368 & 154 & 68 & 54 & 55 & 34 & 498 & 55 & $86 r$ & na & na & na & na \\
\hline AMG496 & 9.31 & 4.87 & 0.659 & 3.30 & 62.82 & & 1.79 & 0.0514 & & & 258 & 292 & 163 & 71 & 53 & 54 & 51 & 468 & 67 & $82 r$ & na & na & na & na \\
\hline AMG497 & 11.63 & 4.73 & 0.657 & 3.36 & & & & & & & 255 & 335 & 174 & 75 & 55 & 49 & 42 & 432 & 73 & & na & na & na & na \\
\hline AMG498 & 7.29 & 5.08 & 0.680 & 3.18 & 64.48 & 16.32 & 1.73 & 0.0543 & 0.92 & & 282 & 177 & 128 & 72 & 46 & 54 & 61 & 444 & 59 & & na & na & na & na \\
\hline AMG499 & 9.09 & 5.00 & 0.674 & 3.09 & 63.53 & 16.22 & 1.28 & 0.0436 & 0.78 & 0.13 & 269 & 218 & 140 & 79 & 54 & 59 & 43 & 554 & 63 & $84 r$ & na & na & na & \\
\hline AMG502 & 19.33 & 4.81 & 0.633 & 2.70 & .05 & 15.04 & 1.50 & 0.0523 & 0.65 & 0.09 & 239 & 378 & 147 & 67 & 62 & 46 & 13 & 363 & 71 & $88 r$ & na & na & na & na \\
\hline AMG503 & 12.92 & 4.86 & 0.696 & 3.12 & 59.51 & 16.58 & 1.36 & 0.0354 & 0.69 & 0.08 & 252 & 399 & 150 & 50 & 61 & 47 & 24 & 406 & 67 & $88 r$ & na & na & na & na \\
\hline AMG504 & 12.93 & 4.77 & 0.653 & 3.01 & & 15.70 & 1.49 & 0.0 & 0.92 & 0.13 & 244 & 307 & 140 & 85 & 44 & 48 & 39 & 463 & 66 & $89 r$ & na & na & na & na \\
\hline 505 & 11.95 & 5.08 & 0.731 & 3.44 & & 16.82 & 2.03 & 0.0 & 0.86 & 0.06 & 274 & 218 & 189 & 82 & 61 & 55 & 33 & 306 & 95 & $97 \mathrm{r}$ & na & na & na & na \\
\hline AMG507 & 11.96 & 5.38 & 0.702 & 3.43 & 59.62 & 16.21 & 1.54 & 0.0429 & 0.79 & 0.18 & 270 & 212 & 172 & 79 & 70 & 52 & 47 & 425 & 70 & $89 r$ & na & na & na & na \\
\hline AMG508 & 11.91 & 5.26 & 0.705 & 3.17 & 59.63 & 16.76 & 1.58 & 0.0396 & 0.64 & 0.15 & 245 & 216 & 157 & 92 & 52 & 54 & 31 & 508 & 69 & $93 r$ & na & na & na & na \\
\hline 509 & 13.08 & 5.37 & 0.715 & 3.37 & 58.40 & 16.31 & 1.68 & 0.0573 & 0.68 & 0.20 & 263 & 226 & 174 & 79 & 62 & 55 & 34 & 441 & 75 & $103 r$ & na & na & na & na \\
\hline AMG510 & 12.82 & 4.92 & 0.663 & 3.40 & 59.30 & 15.83 & 1.94 & 0.0452 & 0.78 & 0.14 & 239 & 263 & 175 & 87 & 54 & 47 & 32 & 407 & 68 & $81 r$ & na & na & na & na \\
\hline AMG511 & 10.25 & 5.47 & 0.732 & 3.02 & 60.72 & 17.26 & 1.48 & 0.0371 & 0.74 & 0.13 & 242 & 205 & 146 & 84 & 63 & 57 & 53 & 518 & 70 & $92 r$ & na & na & na & na \\
\hline AMG512 & 10.90 & 5.37 & 0.714 & 3.38 & 60.39 & 16.51 & 1.61 & 0.0335 & 0.79 & 0.15 & 261 & 212 & 172 & 87 & 60 & 55 & 36 & 469 & 80 & & na & na & na & na \\
\hline AMM 93 & 6.45 & 4.73 & 0.610 & 3.48 & 66.33 & 15.29 & 1.43 & 0.0426 & 1.17 & 0.21 & 281 & 233 & 157 & 87 & 59 & 47 & 21 & 1100 & 70 & $80 r$ & na & na & na & na \\
\hline ASE 18 & 12.27 & 4.37 & 0.559 & 2.86 & 6 & 13.94 & 2.15 & 0.0 & 0.87 & & 238 & 319 & 120 & 75 & 53 & 32 & 33 & 523 & 47 & 83 & 36 & 6 & 31 & 20 \\
\hline ASE 19 & 12.9 & 5.21 & 0.6 & & & & & & & & 223 & 282 & 162 & 61 & 64 & 34 & 38 & 576 & 76 & 91 & 37 & 13 & 44 & 26 \\
\hline & 12.9 & 5.50 & 0.7 & & & & & & & & 250 & 311 & 173 & 00 & 75 & 40 & 41 & 597 & 70 & 94 & 38 & 18 & 36 & 35 \\
\hline & 12.2 & 5.3 & 0.6 & & & & & & & & 242 & 303 & 190 & 0 & 76 & 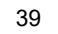 & 40 & & 79 & 89 & 37 & 13 & 40 & 27 \\
\hline 22 & & 4.59 & 0.62 & 3. & & & & & & & 241 & 283 & 172 & 55 & $5 r$ & 35 & 40 & 562 & 53 & 89 & 37 & 8 & 44 & 21 \\
\hline ASE 23 & 11.20 & 5.26 & 0.671 & 3. & & 16 & 1 & & 0.8 & 0.17 & 230 & 330 & 179 & 65 & 71 & 39 & 43 & 570 & 79 & 93 & 34 & 13 & 32 & 22 \\
\hline ASE 24 & 12.68 & 5.45 & 0.693 & 3.5 & & 16. & 1. & & 0.73 & 0.22 & 233 & 260 & 178 & 83 & 66 & 39 & 37 & 563 & 72 & 92 & 39 & 16 & 33 & 20 \\
\hline 25 & 11.95 & 4.86 & 0.633 & 3.44 & 50 & 15 & 7 & 0.0 & 0.81 & 0.33 & 229 & 282 & 165 & 78 & 62 & 38 & 37 & 591 & 66 & 88 & 37 & 11 & 31 & 31 \\
\hline ASE 26 & 11.94 & 5.49 & 0.699 & 3.20 & 83 & 16.83 & & 0.2 & 0.71 & & 243 & 314 & 168 & 62 & 65 & 37 & 41 & 718 & 81 & 87 & 39 & 13 & 38 & 22 \\
\hline ASE 27 & 7.45 & 5.39 & 0.710 & 3.11 & 07 & 17.18 & 1.47 & 0.1 & 0.73 & 0.53 & 270 & 258 & 145 & 65 & 57 & 43 & 48 & 765 & 69 & 106 & 40 & 10 & 68 & 25 \\
\hline ASE 28 & 9.78 & 5.02 & 0.656 & 3.26 & 50 & 15.94 & 1.33 & 0.1317 & 0.88 & 0.31 & 232 & 269 & 168 & 57 & 62 & 39 & 40 & 738 & 70 & 83 & 37 & 13 & 35 & 19 \\
\hline ASE 29 & 12.26 & 4.77 & 0.626 & 2.46 & 61.04 & 15.33 & 1.73 & 0.1199 & 1.28 & 0.21 & 215 & 316 & 137 & 55 & 63 & 34 & 36 & 545 & 60 & 81 & 36 & 10 & 36 & 19 \\
\hline ASE 30 & 11.81 & 4.93 & 0.618 & 3.19 & 61.40 & 15.37 & 1.24 & 0.1974 & 0.74 & 0.33 & 227 & 280 & 159 & 65 & 55 & 35 & 42 & 719 & 65 & 91 & 37 & 15 & 33 & 18 \\
\hline ASE 31 & 8.61 & 5.08 & 0.642 & 2.76 & 64.77 & 15.47 & 1.30 & 0.1421 & 0.76 & 0.28 & 245 & 223 & 116 & 66 & 55 & 41 & 48 & 751 & 67 & 86 & 41 & 10 & 58 & 22 \\
\hline ASE 36 & 15.38 & 4.67 & 0.645 & 3.26 & 58.48 & 15.07 & 1.29 & 0.0365 & 0.89 & 0.11 & 259 & 273 & 172 & 81 & 60 & 33 & 39 & 440 & 75 & 82 & 43 & 15 & 31 & 19 \\
\hline ASE 37 & 10.87 & 4.87 & 0.675 & 3.60 & 61.89 & & & 0.0395 & 1.06 & 0.18 & 285 & 252 & 194 & 85 & 57 & 36 & 36 & 435 & 69 & 98 & 44 & 17 & 21 & 20 \\
\hline FRJ 1 & & 4.82 & 0.64 & & & & & & & & 217 & 238 & 147 & 70 & 54 & 49 & 48 & 817 & 58 & $76 r$ & na & na & na & na \\
\hline FRJ 4 & 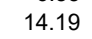 & 4.66 & 0.608 & & & & & & & & 201 & 291 & 179 & 70 & 55 & 52 & 59 & 346 & 70 & $3 r$ & na & na & na & na \\
\hline FRJ 5 & & & & & & & & & & & 10 & & 186 & 70 & & 54 & 41 & 37 & 88 & $82 r$ & na & na & a & na \\
\hline FRJ 6 & & 5 & 0.7 & 3 & 68 & & 2 & & 0 & & 251 & 263 & 155 & 65 & 68 & 51 & 44 & 605 & 74 & $94 r$ & na & na & na & na \\
\hline FRJ 12 & 13 & 5.07 & 0.67 & 3 & 38 & 15 & 18 & 0. & 10 & 0.20 & 220 & 322 & 184 & 80 & 66 & 47 & 37 & 366 & 85 & $87 r$ & na & na & na & na \\
\hline FRJ 18 & 13.20 & 46 & 0.558 & 35 & 75 & 14. & 1.53 & 0.0 & 1.0 & 0.22 & 223 & 346 & 177 & 84 & 60 & 48 & 46 & 395 & 70 & $81 r$ & na & na & na & na \\
\hline VAR 75 & 18.96 & 5.00 & 0.622 & 2.4 & 53.75 & 15.20 & 1.92 & 0.0511 & 1.32 & 0.52 & 201 & 432 & 178 & 60 & 59 & 44 & 44 & 458 & 64 & $85 r$ & na & na & na & na \\
\hline VAR 76 & 6.71 & 4.85 & 0.658 & 3.07 & 65.82 & 16.14 & 1.35 & 0.0508 & 0.66 & 0.48 & 232 & 278 & 141 & 64 & 50 & 52 & 62 & 1101 & 60 & $84 r$ & na & na & na & na \\
\hline VAR 77 & 18.13 & 4.63 & 0.572 & 2.39 & 56.82 & 13.92 & 2.41 & 0.0490 & 0.68 & 0.26 & 211 & 329 & 119 & 65 & 49 & 47 & 35 & 558 & 69 & $71 r$ & na & na & na & na \\
\hline VAR 78 & 9.04 & 4.80 & 0.636 & 2.86 & 63.74 & 15.58 & 1.53 & 0.0341 & 1.35 & 0.27 & 249 & 286 & 161 & 55 & 55 & 48 & 66 & 600 & 74 & $83 r$ & na & na & na & na \\
\hline VAR 79 & 6.44 & 4.88 & 0.657 & 3.49 & 65.54 & 16.10 & 1.53 & 0.0435 & 0.81 & 0.34 & 239 & 266 & 189 & 71 & 47 & 53 & 80 & 630 & 70 & $83 r$ & na & na & na & na \\
\hline VAR 80 & 11.93 & 4.78 & 0.624 & 3.10 & 61.25 & 15.20 & 1.55 & 0.0483 & 1.04 & 0.30 & 222 & 282 & 178 & 70 & 59 & 47 & 70 & 570 & 63 & $81 r$ & na & na & na & na \\
\hline VAR 81 & 12.54 & 4.86 & 0.639 & 3.3 & 59.80 & 15.54 & 1.65 & 0.0505 & 1.02 & 0.34 & 251 & 286 & 209 & 73 & 58 & 46 & 70 & 452 & 69 & $83 r$ & na & na & na & na \\
\hline VAR 82 & 12.88 & 5.00 & 0.642 & 3.2 & 59.80 & 15.35 & 1.42 & 0.0646 & 1.03 & 0.35 & 244 & 266 & 192 & 72 & 51 & 48 & 58 & 525 & 79 & $78 r$ & na & na & na & na \\
\hline VAR 83 & 8.2 & $508-2 x$ & 0.679 & 3.6 & 63. & 16 & 1.46 & 0.0477 & 0.91 & 0.23 & 259 & 253 & 196 & 97 & 44 & 53 & 84 & 635 & 73 & $82 r$ & na & na & na & na \\
\hline VAR 84 & 11 & & 0.668 & 3.4 & & & & & & & 244 & 262 & 200 & 82 & 57 & 44 & 63 & 477 & 67 & $85 r$ & na & na & na & na \\
\hline VAR 85 & 1 & & 0.644 & & & & & & & & 252 & 293 & 192 & 68 & 48 & 41 & 49 & 491 & 68 & $82 r$ & na & na & na & na \\
\hline VAR 86 & 10 & & & & & & & & & & 260 & 281 & 217 & 72 & 64 & 49 & 64 & & 76 & $83 r$ & na & na & na & na \\
\hline VAR 93 & 13.78 & & 0.579 & & & & & & & & 221 & 325 & 190 & 70 & 41 & 43 & 54 & & 66 & $76 r$ & na & na & na & na \\
\hline VAR 94 & 16.66 & 5.46 & 0.687 & 2.60 & 55.18 & 16.52 & 1.49 & 0.0740 & 0.64 & 0.51 & 234 & 431 & 162 & 58 & 58 & 44 & 53 & 515 & 94 & $84 r$ & na & na & na & na \\
\hline VAR 95 & 11.61 & 4.42 & 0.581 & 3.46 & 62.19 & 14.60 & 1.84 & 0.0370 & 0.91 & 0.20 & 219 & 271 & 196 & 77 & 48 & 44 & 53 & 370 & 69 & $69 r$ & na & na & na & na \\
\hline VAR 96 & 11.78 & 4.42 & 0.581 & 2.81 & 62.93 & 14.08 & 2.16 & 0.0392 & 0.82 & 0.22 & 241 & 306 & 134 & 79 & 50 & 55 & 44 & 522 & 57 & $77 r$ & na & na & na & na \\
\hline VAR 97 & 11.64 & 4.65 & 0.593 & 3.28 & 62.30 & 14.56 & 1.53 & 0.0680 & 0.97 & 0.26 & 234 & 253 & 188 & 80 & 55 & 44 & 67 & 392 & 72 & $87 r$ & na & na & na & na \\
\hline
\end{tabular}




\begin{tabular}{|c|c|c|c|c|c|c|c|c|c|c|c|c|c|c|c|c|c|c|c|c|c|c|c|c|}
\hline \multicolumn{25}{|c|}{ Jardin d'Ariane, amphorettes $(n=4)$} \\
\hline Nom & $\mathrm{CaO}$ & $\mathrm{Fe}_{2} \mathrm{O}_{3}$ & $\mathrm{TiO}_{2}$ & $\mathrm{~K}_{2} \mathrm{O}$ & $\mathrm{SiO}_{2}$ & $\mathrm{Al}_{2} \mathrm{O}_{3}$ & $\mathrm{MgO}$ & $\mathrm{MnO}$ & $\mathrm{Na}_{2} \mathrm{O}$ & $\mathrm{P}_{2} \mathrm{O}_{5}$ & $\mathrm{Zr}$ & $\mathrm{Sr}$ & $\mathrm{Rb}$ & $\mathrm{Zn}$ & $\mathrm{Cr}$ & $\mathrm{Ni}$ & La & $\mathrm{Ba}$ & V & $\mathrm{Ce}$ & Y & Th & $\mathrm{Pb}$ & $\mathrm{Cu}$ \\
\hline ASE 32 & 2.50 & 5.74 & 0.781 & 3.57 & 67.30 & 17.43 & 1.36 & 0.0659 & 0.93 & 0.14 & 280 & 132 & 227 & 95 & 70 & 45 & 41 & 547 & 95 & 96 & 43 & 15 & 37 & 25 \\
\hline ASE 33 & 0.88 & 6.20 & 0.856 & 3.60 & 66.66 & 19.27 & 1.33 & 0.0338 & 0.87 & 0.11 & 300 & 105 & 196 & 140 & 64 & 70 & 47 & 569 & 94 & 91 & 43 & 15 & 50 & 26 \\
\hline ASE 34 & 1.41 & 5.88 & 0.797 & 3.51 & 67.67 & 17.88 & 1.56 & 0.1626 & 0.84 & 0.12 & 278 & 105 & 220 & 121 & 62 & 50 & 39 & 486 & 99 & 104 & 39 & 17 & 34 & 29 \\
\hline ASE 35 & 1.12 & 6.13 & 0.814 & 3.49 & 67.57 & 18.20 & 1.51 & 0.0483 & 0.83 & 0.12 & 280 & 94 & 203 & 118 & 67 & 53 & 49 & 442 & 93 & 103 & 41 & 13 & 52 & 20 \\
\hline
\end{tabular}

\begin{tabular}{|c|c|c|c|c|c|c|c|c|c|c|c|c|c|c|c|c|c|c|c|c|c|c|c|c|}
\hline \multicolumn{25}{|c|}{ Lorgues $(n=11)$} \\
\hline Nom & $\mathrm{CaO}$ & $\mathrm{Fe}_{2} \mathrm{O}_{3}$ & $\mathrm{TiO}_{2}$ & $\mathrm{~K}_{2} \mathrm{O}$ & $\mathrm{SiO}_{2}$ & $\mathrm{Al}_{2} \mathrm{O}_{3}$ & $\mathrm{MgO}$ & $\mathrm{MnO}$ & $\mathrm{Na}_{2} \mathrm{O}$ & $\mathrm{P}_{2} \mathrm{O}_{5}$ & $\mathrm{Zr}$ & $\mathrm{Sr}$ & $\mathrm{Rb}$ & $\mathrm{Zn}$ & $\mathrm{Cr}$ & $\mathrm{Ni}$ & La & $\mathrm{Ba}$ & V & $\mathrm{Ce}$ & $Y$ & Th & $\mathrm{Pb}$ & $\mathrm{Cu}$ \\
\hline VAR 6 & 6.88 & 7.16 & 0.870 & 3.51 & 57.15 & 21.10 & 2.90 & 0.0995 & 0.00 & 0.10 & 215 & 96 & 150 & 98 & 109 & 58 & 49 & 711 & 108 & 97 & na & na & na & na \\
\hline VAR 9 & 9.10 & 6.99 & 0.760 & 3.64 & 54.29 & 21.64 & 3.21 & 0.0727 & 0.00 & 0.10 & 166 & 126 & 180 & 104 & 107 & 52 & 32 & 571 & 110 & 87 & na & na & na & na \\
\hline VAR 10 & 6.90 & 7.30 & 0.870 & 3.29 & 58.27 & 20.46 & 2.54 & 0.0639 & 0.00 & 0.11 & 216 & 95 & 151 & 95 & 104 & 49 & 42 & 647 & 114 & 97 & na & na & na & na \\
\hline VAR 45 & 4.01 & 7.30 & 0.850 & 4.07 & 56.67 & 23.30 & 2.89 & 0.0876 & 0.49 & 0.10 & 241 & 90 & 160 & 100 & 103 & 54 & 77 & 727 & 110 & 92 & na & na & na & na \\
\hline VAR 46 & 3.00 & 7.39 & 0.900 & 4.19 & 58.04 & 22.66 & 2.99 & 0.0859 & 0.42 & 0.11 & 252 & 90 & 179 & 115 & 102 & 59 & 55 & 731 & 118 & 100 & na & na & na & na \\
\hline VAR 65 & 7.05 & 6.79 & 0.840 & 3.55 & 57.88 & 20.30 & 2.87 & 0.0921 & 0.28 & 0.15 & 228 & 104 & 163 & 93 & 91 & 57 & 39 & 673 & 97 & 89 & na & na & na & na \\
\hline VAR 67 & 7.94 & 6.83 & 0.830 & 3.44 & 55.47 & 21.49 & 3.24 & 0.0712 & 0.25 & 0.22 & 227 & 102 & 159 & 95 & 91 & 60 & 53 & 702 & 103 & 112 & na & na & na & na \\
\hline VAR 68 & 9.10 & 6.31 & 0.790 & 3.50 & 55.75 & 20.34 & 3.52 & 0.0755 & 0.30 & 0.12 & 214 & 98 & 168 & 86 & 87 & 50 & 64 & 660 & 98 & 99 & na & na & na & na \\
\hline VAR 70 & 3.23 & 6.62 & 0.880 & 3.49 & 62.40 & 20.02 & 2.66 & 0.0767 & 0.29 & 0.11 & 269 & 86 & 147 & 101 & 81 & 55 & 75 & 652 & 110 & 107 & na & na & na & na \\
\hline VAR 71 & 13.15 & 6.98 & 0.750 & 3.04 & 52.30 & 20.50 & 2.57 & 0.0600 & 0.36 & 0.09 & 167 & 110 & 167 & 97 & 96 & 57 & 50 & 592 & 119 & 78 & na & na & na & na \\
\hline VAR 72 & 10.85 & 7.12 & 0.770 & 3.22 & 53.85 & 20.99 & 2.52 & 0.0569 & 0.31 & 0.10 & 168 & 111 & 166 & 94 & 99 & 63 & 32 & 642 & 97 & 76 & na & na & na & na \\
\hline
\end{tabular}

\begin{tabular}{|c|c|c|c|c|c|c|c|c|c|c|c|c|c|c|c|c|c|c|c|c|c|c|c|c|}
\hline \multicolumn{25}{|c|}{ Fox-Amphoux $(n=5)$} \\
\hline Nom & $\mathrm{CaO}$ & $\mathrm{Fe}_{2} \mathrm{O}_{3}$ & $\mathrm{TiO}_{2}$ & $\mathrm{~K}_{2} \mathrm{O}$ & $\mathrm{SiO}_{2}$ & $\mathrm{Al}_{2} \mathrm{O}_{3}$ & $\mathrm{MgO}$ & $\mathrm{MnO}$ & $\mathrm{Na}_{2} \mathrm{O}$ & $\mathrm{P}_{2} \mathrm{O}_{5}$ & $\mathrm{Zr}$ & $\mathrm{Sr}$ & $\mathrm{Rb}$ & $\mathrm{Zn}$ & $\mathrm{Cr}$ & $\mathrm{Ni}$ & La & $\mathrm{Ba}$ & V & $\mathrm{Ce}$ & $\mathrm{Y}$ & Th & $\mathrm{Pb}$ & $\mathrm{Cu}$ \\
\hline ASE135 & 7.07 & 7.09 & 0.767 & 4.33 & 56.41 & 20.95 & 2.82 & 0.1107 & 0.08 & 0.18 & 169 & 265 & 226 & 101 & 77 & 45 & 43 & 736 & 115 & 113 & 34 & 14 & 40 & 27 \\
\hline ASE136 & 5.64 & 7.66 & 0.785 & 4.60 & 55.79 & 21.81 & 2.99 & 0.1019 & 0.13 & 0.28 & 158 & 245 & 243 & 116 & 85 & 49 & 51 & 877 & 116 & 116 & 31 & 15 & 51 & 26 \\
\hline ASE137 & 6.05 & 7.85 & 0.763 & 4.40 & 56.35 & 20.71 & 2.68 & 0.1766 & 0.09 & 0.68 & 171 & 278 & 224 & 213 & 78 & 44 & 46 & 1157 & 112 & 113 & 34 & 15 & 45 & 48 \\
\hline ASE138 & 6.03 & 7.62 & 0.766 & 4.54 & 55.67 & 21.79 & 2.96 & 0.0998 & 0.11 & 0.22 & 165 & 224 & 240 & 103 & 78 & 47 & 44 & 778 & 95 & 108 & 32 & 15 & 30 & 25 \\
\hline ASE139 & 8.23 & 7.31 & 0.746 & 4.48 & 54.10 & 21.23 & 3.22 & 0.1172 & 0.14 & 0.22 & 153 & 339 & 239 & 113 & 80 & 46 & 44 & 779 & 99 & 110 & 33 & 14 & 39 & 40 \\
\hline ASE140 & 6.58 & 7.28 & 0.768 & 4.40 & 55.31 & 21.37 & 3.12 & 0.1051 & 0.17 & 0.69 & 162 & 312 & 233 & 113 & 86 & 47 & 43 & 846 & 112 & 106 & 34 & 15 & 39 & 30 \\
\hline \multicolumn{25}{|c|}{ La Celle Revou $(n=3)$} \\
\hline Nom & $\mathrm{CaO}$ & $\mathrm{Fe}_{2} \mathrm{O}_{3}$ & $\mathrm{TiO}_{2}$ & $\mathrm{~K}_{2} \mathrm{O}$ & $\mathrm{SiO}_{2}$ & $\mathrm{Al}_{2} \mathrm{O}_{3}$ & $\mathrm{MgO}$ & $\mathrm{MnO}$ & $\mathrm{Na}_{2} \mathrm{O}$ & $\mathrm{P}_{2} \mathrm{O}_{5}$ & $\mathrm{Zr}$ & $\mathrm{Sr}$ & $\mathrm{Rb}$ & $\mathrm{Zn}$ & $\mathrm{Cr}$ & $\mathrm{Ni}$ & La & $\mathrm{Ba}$ & V & $\mathrm{Ce}$ & $\mathrm{Y}$ & Th & $\mathrm{Pb}$ & $\mathrm{Cu}$ \\
\hline VAR 11 & 10.43 & 6.72 & 0.770 & 2.54 & 58.86 & 18.28 & 1.99 & 0.0937 & 0.00 & 0.12 & 232 & 186 & 115 & 100 & 107 & 67 & 34 & 462 & 87 & 99 & na & na & na & na \\
\hline VAR 12 & 8.63 & 5.95 & 0.950 & 2.84 & 61.64 & 18.22 & 1.37 & 0.0796 & 0.00 & 0.13 & 283 & 143 & 107 & 95 & 108 & 61 & 71 & 424 & 106 & 96 & na & na & na & na \\
\hline VAR 13 & 11.21 & 6.42 & 0.840 & 2.40 & 58.68 & 18.85 & 1.19 & 0.0914 & 0.00 & 0.12 & 239 & 195 & 122 & 86 & 119 & 71 & 91 & 389 & 121 & 92 & na & na & na & na \\
\hline
\end{tabular}

\begin{tabular}{|c|c|c|c|c|c|c|c|c|c|c|c|c|c|c|c|c|c|c|c|c|c|c|c|c|}
\hline \multicolumn{25}{|c|}{ Bras $(n=3)$} \\
\hline Nom & $\mathrm{CaO}$ & $\mathrm{Fe}_{2} \mathrm{O}_{3}$ & $\mathrm{TiO}_{2}$ & $\mathrm{~K}_{2} \mathrm{O}$ & $\mathrm{SiO}_{2}$ & $\mathrm{Al}_{2} \mathrm{O}_{3}$ & $\mathrm{MgO}$ & $\mathrm{MnO}$ & $\mathrm{Na}_{2} \mathrm{O}$ & $\mathrm{P}_{2} \mathrm{O}_{5}$ & $\mathrm{Zr}$ & $\mathrm{Sr}$ & $\mathrm{Rb}$ & $\mathrm{Zn}$ & $\mathrm{Cr}$ & $\mathrm{Ni}$ & $\mathrm{La}$ & $\mathrm{Ba}$ & V & $\mathrm{Ce}$ & $\mathrm{Y}$ & Th & $\mathrm{Pb}$ & $\mathrm{Cu}$ \\
\hline AMM108 & 9.15 & 7.43 & 0.710 & 3.74 & 53.04 & 20.63 & 4.50 & 0.0838 & 0.31 & 0.16 & 180 & 141 & 178 & 105 & 93 & 45 & 61 & 838 & 106 & 100 & na & na & na & na \\
\hline AMM109 & 10.90 & 6.73 & 0.680 & 3.47 & 54.03 & 18.66 & 4.77 & 0.0762 & 0.31 & 0.17 & 186 & 154 & 158 & 96 & 85 & 42 & 0 & 766 & 100 & 101 & na & na & na & na \\
\hline AMM110 & 11.83 & 7.49 & 0.760 & 2.56 & 52.31 & 20.12 & 4.14 & 0.1155 & 0.30 & 0.16 & 207 & 147 & 119 & 92 & 95 & 48 & 5 & 762 & 89 & 104 & na & na & na & na \\
\hline
\end{tabular}

\begin{tabular}{|c|c|c|c|c|c|c|c|c|c|c|c|c|c|c|c|c|c|c|c|c|c|c|c|c|}
\hline \multicolumn{25}{|c|}{ Marseille } \\
\hline Nom & $\mathrm{CaO}$ & $\mathrm{Fe}_{2} \mathrm{O}_{3}$ & $\mathrm{TiO}_{2}$ & $\mathrm{~K}_{2} \mathrm{O}$ & $\mathrm{SiO}_{2}$ & $\mathrm{Al}_{2} \mathrm{O}_{3}$ & $\mathrm{MgO}$ & $\mathrm{MnO}$ & $\mathrm{Na}_{2} \mathrm{O}$ & $\mathrm{P}_{2} \mathrm{O}_{5}$ & $\mathrm{Zr}$ & $\mathrm{Sr}$ & $\mathrm{Rb}$ & $\mathrm{Zn}$ & $\mathrm{Cr}$ & $\mathrm{Ni}$ & La & $\mathrm{Ba}$ & V & $\mathrm{Ce}$ & $\mathrm{Y}$ & Th & $\mathrm{Pb}$ & $\mathrm{Cu}$ \\
\hline \multicolumn{25}{|c|}{ Carmes - $1(n=24)$} \\
\hline AMM 36 & 21.39 & 5.63 & 0.670 & 3.53 & 49.69 & 15.31 & 3.31 & 0.0797 & 0.02 & 0.17 & 157 & 407 & 151 & 78 & 85 & 43 & 63 & 411 & 96 & 93 & na & na & na & na \\
\hline AMM 38 & 21.53 & 5.55 & 0.670 & 3.60 & 49.42 & 15.36 & 3.28 & 0.0791 & 0.18 & 0.12 & 144 & 402 & 149 & 95 & 89 & 44 & 78 & 474 & 96 & 91 & na & na & na & na \\
\hline AMM 31 & 22.55 & 5.64 & 0.660 & 3.56 & 48.56 & 15.41 & 3.16 & 0.0800 & 0.04 & 0.13 & 152 & 452 & 151 & 87 & 87 & 50 & 46 & 456 & 106 & 94 & na & na & na & na \\
\hline AMM 37 & 22.67 & 5.65 & 0.670 & 3.50 & 47.65 & 15.31 & 4.03 & 0.0861 & 0.04 & 0.14 & 152 & 431 & 157 & 80 & 92 & 47 & 75 & 857 & 106 & 83 & 3 na & na & na & na \\
\hline AMM 35 & 21.29 & 5.56 & 0.660 & 3.66 & 48.76 & 15.41 & 4.22 & 0.0750 & 0.05 & 0.12 & 155 & 449 & 145 & 90 & 78 & 46 & 0 & 423 & 104 & 86 & na & na & na & na \\
\hline AMM 30 & 21.31 & 6.28 & 0.700 & 3.43 & 47.64 & 16.67 & 3.41 & 0.0843 & 0.11 & 0.16 & 143 & 351 & 164 & 91 & 87 & 52 & 0 & 468 & 106 & 93 & na & na & na & na \\
\hline AMM 55 & 21.17 & 5.62 & 0.660 & 3.29 & 48.35 & 16.42 & 4.12 & 0.0806 & 0.00 & 0.10 & 146 & 359 & 143 & 96 & 88 & 50 & 67 & 425 & 102 & 93 & na & na & na & na \\
\hline AMM 40 & 21.39 & 5.71 & 0.660 & 3.28 & 48.49 & 16.33 & 3.68 & 0.0836 & 0.06 & 0.12 & 149 & 347 & 133 & 89 & 81 & 46 & 90 & 427 & 87 & 81 & na & na & na & : \\
\hline AMM 48 & 22.59 & 5.42 & 0.640 & 3.02 & 48.75 & & 3.56 & 0.0851 & 0.09 & 0.12 & 154 & 369 & 134 & 76 & 80 & 45 & 65 & 401 & 92 & 87 & na & na & a & m \\
\hline AMM 49 & 19.16 & 5.74 & 0.680 & 3.17 & 50.45 & 16.75 & 3.64 & 0.0820 & 0.00 & 0.14 & 138 & 381 & 143 & 81 & 87 & 48 & 68 & 424 & 93 & 86 & na & na & na & na \\
\hline AMM 39 & 23.33 & 5.16 & 0.620 & 2.65 & 48.26 & 14.86 & 4.19 & 0.0723 & 0.54 & 0.11 & 149 & 384 & 127 & 80 & 79 & 40 & 89 & 420 & 89 & 84 & na & na & na & na \\
\hline AMM 42 & 22.46 & 5.02 & 0.650 & 3.01 & 49.57 & 5.43 & 3.42 & 0.0788 & 0.04 & 0.11 & 159 & 345 & 147 & 80 & 80 & 38 & 30 & 433 & 81 & 91 & na & na & na & na \\
\hline AMM 41 & 22.78 & 5.11 & 0.630 & 3.78 & 48.49 & 15.45 & 3.38 & 0.0754 & 0.00 & 0.11 & 153 & 311 & 153 & 85 & 85 & 42 & 53 & 441 & 99 & 84 & na & na & na & na \\
\hline AMM 52 & 18.98 & 5.51 & 0.660 & 3.33 & 53.05 & 15.10 & 2.96 & 0.0811 & 0.01 & 0.12 & 176 & 341 & 137 & 86 & 80 & 45 & 92 & 428 & 96 & 93 & na & na & na & na \\
\hline AMM 54 & 19.10 & 5.34 & 0.660 & 3.22 & 52.53 & 15.24 & 3.51 & 0.0792 & 0.00 & 0.13 & 170 & 312 & 140 & 81 & 73 & 42 & 57 & 495 & 97 & 86 & na & na & na & na \\
\hline AMM 53 & 21.53 & 5.48 & 0.660 & 3.03 & 50.26 & 15.10 & 3.55 & 0.0736 & 0.01 & 0.12 & 172 & 332 & 137 & 83 & 78 & 49 & 32 & 420 & 89 & 92 & na & na & na & na \\
\hline AMM 46 & 20.18 & 4.75 & 0.650 & 3.13 & 54.12 & 14.24 & 2.56 & 0.0801 & 0.00 & 0.11 & 173 & 346 & 142 & 71 & 75 & 46 & 31 & 371 & 96 & 87 & na & na & na & na \\
\hline AMM 33 & 18.91 & 5.26 & 0.640 & 3.52 & 52.11 & 15.26 & 3.52 & 0.0725 & 0.38 & 0.12 & 154 & 354 & 153 & 79 & 75 & 47 & 70 & 500 & 90 & 80 & na & na & na & na \\
\hline AMM 43 & 22.29 & 4.61 & 0.610 & 2.72 & 52.67 & 13.24 & 3.37 & 0.0757 & 0.06 & 0.13 & 168 & 433 & 129 & 71 & 71 & 45 & 15 & 538 & 83 & 86 & na & na & na & na \\
\hline AMM 47 & 20.38 & 5.81 & 0.670 & 2.43 & 50.34 & 14.68 & 4.50 & 0.08 & 0.51 & 0.38 & 179 & 485 & 144 & 92 & 81 & 44 & 50 & 489 & 90 & 93 & na & na & na & na \\
\hline AMM 50 & 21.70 & 5.50 & 0.670 & 2.31 & 48. & 16 & 4.18 & 0.0 & 0. & 0.13 & 152 & 468 & 165 & 85 & 93 & 44 & 54 & 550 & 98 & 93 & na & na & a & na \\
\hline AMM 51 & 17.34 & 6.32 & 0.750 & 3.5 & 49. & & 3.62 & 0.0 & 0. & & 162 & 423 & 160 & 114 & 87 & 46 & 93 & 520 & 100 & 97 & na & na & a & na \\
\hline AMM 56 & 18.89 & 5.91 & 0.710 & 2.9 & 51. & & 2.78 & & 0. & & 167 & 441 & 143 & 95 & 83 & 48 & 94 & 697 & 96 & 98 & na & na & la & าa \\
\hline AMM 45 & 22.32 & 6.03 & 0.690 & 3.37 & 47.29 & 16.32 & 3.34 & 0.0901 & 0.13 & 0.16 & 156 & 420 & 152 & 93 & 106 & 46 & 108 & 809 & 123 & 91 & na & na & na & ( \\
\hline \multicolumn{25}{|c|}{ Carmes - $2(n=3)$} \\
\hline ASE 72 & 17.16 & 5.69 & 0.654 & 2.8 & 5 & & 5 & & 0.1 & 0.20 & 195 & 411 & 61 & 74 & 67 & 35 & 33 & 584 & 77 & 91 & 33 & 12 & 31 & 28 \\
\hline ASE 77 & 15.57 & 5.64 & 0.649 & 2.38 & 53.46 & 15 & 6.67 & 0.0784 & 0.08 & 0.23 & 187 & 384 & 82 & 80 & 49 & 39 & 38 & 783 & 81 & 88 & 33 & 10 & 26 & 37 \\
\hline ASE 75 & 22.51 & 5.27 & 0.609 & 2.41 & 49.49 & 13.79 & 5.22 & 0.0719 & 0.17 & 0.26 & 177 & 570 & 51 & 74 & 53 & 28 & 34 & 708 & 90 & 85 & 33 & 12 & 25 & 26 \\
\hline \multicolumn{25}{|c|}{ Carmes - $3(n=6)$} \\
\hline ASE 68 & 29.85 & 4.73 & 0.558 & 2.62 & 46.16 & 12.56 & 3.02 & 0.0463 & 0.16 & 0.17 & 167 & 384 & 86 & 71 & 62 & 23 & 33 & 317 & 89 & 67 & 29 & 9 & 21 & 26 \\
\hline ASE 69 & 29.51 & 5.20 & 0.575 & 2.74 & 44.50 & 13.48 & 3.46 & 0.0440 & 0.19 & 0.17 & 166 & 360 & 96 & 78 & 69 & 27 & 31 & 293 & 78 & 70 & 30 & 14 & 12 & 27 \\
\hline ASE 70 & 28.04 & 4.48 & 0.545 & 2.37 & 49.46 & 11.38 & 3.20 & 0.0514 & 0.19 & 0.16 & 193 & 352 & 66 & 63 & 53 & 23 & 31 & 242 & 54 & 73 & 29 & 12 & 23 & 33 \\
\hline ASE 73 & 24.59 & 5.49 & 0.627 & 1.77 & 48.50 & 15.26 & 2.74 & 0.0553 & 0.48 & 0.33 & 166 & 365 & 94 & 62 & 59 & 28 & 28 & 563 & 55 & 77 & 33 & 15 & 22 & 27 \\
\hline ASE 76 & 23.65 & 5.60 & 0.640 & 1.51 & 49.37 & 15.57 & 2.45 & 0.0602 & 0.54 & 0.42 & 179 & 502 & 94 & 67 & 66 & 30 & 36 & 677 & 50 & 77 & 31 & 14 & 17 & 24 \\
\hline ASE 71 & 25.93 & 5.45 & 0.621 & 1.34 & 46.33 & 15.06 & 3.86 & 0.0420 & 1.06 & 0.18 & 164 & 330 & 116 & 76 & 79 & 33 & 33 & 256 & 82 & 80 & 32 & 15 & 10 & 34 \\
\hline \multicolumn{25}{|c|}{$\begin{array}{ll}0.10 \\
104\end{array}$} \\
\hline ASE 78 & 9.31 & 8 & 0.827 & 37 & 53.02 & & 2 & & 0 & 0.31 & 175 & 304 & 175 & 106 & 86 & 46 & 48 & 759 & 113 & 99 & 34 & 17 & 40 & 39 \\
\hline ASE 79 & 14.21 & 6.9 & 0.780 & 1.4 & & & & & & & 199 & 384 & 119 & 54 & 89 & 39 & 42 & 526 & 110 & 97 & 34 & 15 & 37 & 44 \\
\hline ASE 80 & & 8.15 & 0.844 & 3.2 & & & & & & 0.44 & 184 & 333 & 151 & 81 & 91 & 47 & 43 & 753 & 113 & 95 & 31 & 0 & 121 & 40 \\
\hline ASE 81 & 16.80 & 5.97 & 0.671 & 1.24 & 52.11 & 15.45 & 6.91 & 0.0559 & 0.20 & 0.41 & 189 & 709 & 34 & 80 & 46 & 48 & 39 & 347 & 69 & 101 & 33 & 6 & 41 & 69 \\
\hline ASE 82 & 11.97 & 7.81 & 0.827 & 1.65 & 49.35 & 22.66 & 4.04 & 0.0789 & 0.87 & 0.53 & 175 & 402 & 112 & 88 & 91 & 51 & 44 & 738 & 110 & 101 & 33 & 0 & 116 & 48 \\
\hline
\end{tabular}




\begin{tabular}{|c|c|c|c|c|c|c|c|c|c|c|c|c|c|c|c|c|c|c|c|c|c|c|c|c|}
\hline \multicolumn{25}{|c|}{ Aix-en-Provence } \\
\hline Nom & $\mathrm{CaO}$ & $\mathrm{Fe}_{2} \mathrm{O}_{3}$ & $\mathrm{TiO}_{2}$ & $\mathrm{~K}_{2} \mathrm{O}$ & $\mathrm{SiO}_{2}$ & $\mathrm{Al}_{2} \mathrm{O}_{3}$ & $\mathrm{MgO}$ & $\mathrm{MnO}$ & $\mathrm{Na}_{2} \mathrm{O}$ & $\mathrm{P}_{2} \mathrm{O}_{5}$ & $\mathrm{Zr}$ & $\mathrm{Sr}$ & $\mathrm{Rb}$ & $\mathrm{Zn}$ & $\mathrm{Cr}$ & $\mathrm{Ni}$ & La & $\mathrm{Ba}$ & V & $\mathrm{Ce}$ & Y & Th & $\mathrm{Pb}$ & $\mathrm{Cu}$ \\
\hline \multicolumn{25}{|c|}{ République $(n=11)$} \\
\hline ASE 47 & 23.36 & 5.25 & 0.617 & 1.14 & 51.08 & 13.90 & 3.08 & 0.0969 & 1.14 & 0.13 & 121 & 830 & 112 & 78 & 131 & 93 & 29 & 268 & 112 & 74 & 26 & 8 & 13 & 42 \\
\hline ASE $48^{*}$ & 13.83 & 6.63 & 0.727 & 3.86 & 54.13 & 18.21 & 1.87 & 0.0855 & 0.33 & 0.13 & 183 & 384 & 185 & 77 & 80 & 41 & 45 & 617 & 108 & 97 & 33 & 12 & 34 & 28 \\
\hline ASE 49* & 20.94 & 4.79 & 0.583 & 2.47 & 52.47 & 14.05 & 4.03 & 0.0370 & 0.2 & 0.15 & 136 & 1358 & 97 & 77 & 75 & 40 & 31 & 499 & 118 & 78 & 25 & 10 & 23 & 28 \\
\hline ASE 50 & 28.44 & 5.16 & 0.542 & 2.5 & 46. & 13.36 & 2.60 & 0.0885 & 0.25 & 0.18 & 108 & 797 & 100 & 76 & 101 & 76 & 25 & 347 & 79 & 67 & 23 & 5 & 19 & 36 \\
\hline ASE 51 & 30.85 & 5.22 & 0.559 & 0.86 & 45.70 & 13.02 & 2.39 & 0.0710 & 1.02 & 0.15 & 112 & 807 & 59 & 63 & 111 & 66 & 26 & 260 & 108 & 68 & 23 & 11 & 10 & 28 \\
\hline ASE 52 & 20.65 & 5.40 & 0.617 & 2.87 & 52.45 & 14.14 & 3.15 & 0.1233 & 0.24 & 0.15 & 126 & 662 & 115 & 84 & 99 & 97 & 31 & 539 & 101 & 75 & 26 & 12 & 30 & 38 \\
\hline ASE 53 & 25.30 & 5.22 & 0.564 & 2.22 & 49.68 & 13.64 & 2.55 & 0.0778 & 0.41 & 0.16 & 114 & 806 & 94 & 71 & 119 & 75 & 28 & 357 & 86 & 68 & 23 & 9 & 11 & 33 \\
\hline ASE 54 & 29.94 & 4.67 & 0.527 & 1.40 & 48.01 & 11.88 & 2.49 & 0.0656 & 0.66 & 0.18 & 136 & 827 & 96 & 65 & 96 & 58 & 23 & 296 & 66 & 66 & 24 & 11 & 13 & 24 \\
\hline ASE 55 & 27.48 & 4.76 & 0.575 & 2.32 & 47.81 & 12.69 & 3.53 & 0.1238 & 0.40 & 0.13 & 114 & 736 & 92 & 76 & 117 & 73 & 27 & 299 & 94 & 72 & 26 & 9 & 13 & 39 \\
\hline ASE 56 & 26.11 & 5.32 & 0.566 & 2.00 & 48.87 & 13.96 & 2.30 & 0.0806 & 0.44 & 0.15 & 107 & 874 & 91 & 70 & 128 & 80 & 23 & 403 & 88 & 67 & 22 & 11 & 14 & 32 \\
\hline \multicolumn{24}{|c|}{ Monclar $(n=6)$} & 26 \\
\hline ASE 41 & 15.96 & 6.84 & 0.662 & 0.46 & 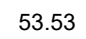 & 16.21 & 4.35 & 0.1023 & 1.7 & 0.28 & 114 & 669 & 73 & 82 & 145 & 109 & 31 & 252 & 131 & 89 & 24 & 8 & 31 & 40 \\
\hline ASE 42 & 14.33 & 7.13 & 0.718 & 1.43 & 53.58 & 17.42 & 3.79 & 0.0960 & 1.02 & 0.29 & 120 & 602 & 125 & 69 & 155 & 126 & 31 & 442 & 108 & 80 & 24 & 8 & 45 & 49 \\
\hline ASE 43* & 14.73 & 7.31 & 0.772 & 2.72 & 51.00 & 18.71 & 2.92 & 0.1179 & 0.43 & 1.06 & 195 & 815 & 113 & 85 & 81 & 47 & 41 & 592 & 85 & 99 & 37 & 10 & 61 & 42 \\
\hline ASE $44^{*}$ & 10.79 & 7.74 & 0.742 & 3.00 & 52.18 & 20.95 & 3.22 & 0.0885 & 0.24 & 0.83 & 176 & 497 & 139 & 97 & 91 & 50 & 46 & 702 & 89 & 102 & 36 & 0 & 124 & 40 \\
\hline ASE 45 & 16.70 & 6.72 & 0.671 & 0.6 & 53.30 & & 3.79 & 0.0915 & 1.49 & 0.25 & 116 & 643 & 95 & 69 & 145 & 108 & 36 & 354 & 123 & 76 & 26 & 7 & 33 & 48 \\
\hline ASE 46 & 19.20 & 6.41 & 0.656 & 1.25 & 52.10 & 15.50 & 3.67 & 0.1047 & 0.44 & 0.45 & 118 & 746 & 61 & 64 & 130 & 103 & 33 & 483 & 105 & 76 & 26 & 13 & 34 & 39 \\
\hline
\end{tabular}

\begin{tabular}{|c|c|c|c|c|c|c|c|c|c|c|c|c|c|c|c|c|c|c|c|c|c|c|c|c|}
\hline \multicolumn{25}{|l|}{ Velaux } \\
\hline Nom & $\mathrm{CaO}$ & $\mathrm{Fe}_{2} \mathrm{O}_{3}$ & $\mathrm{TiO}_{2}$ & $\mathrm{~K}_{2} \mathrm{O}$ & $\mathrm{SiO}_{2}$ & $\mathrm{Al}_{2} \mathrm{O}_{3}$ & $\mathrm{MgO}$ & $\mathrm{MnO}$ & $\mathrm{Na}_{2} \mathrm{O}$ & $\mathrm{P}_{2} \mathrm{O}_{5}$ & $\mathrm{Zr}$ & $\mathrm{Sr}$ & $\mathrm{Rb}$ & $\mathrm{Zn}$ & $\mathrm{Cr}$ & $\mathrm{Ni}$ & $\mathrm{La}$ & $\mathrm{Ba}$ & V & $\mathrm{Ce}$ & Y & Th & $\mathrm{Pb}$ & $\mathrm{Cu}$ \\
\hline \multicolumn{25}{|c|}{ Moulin du Pont $(n=19)$} \\
\hline ASE 91 & 11.33 & 6.92 & 0.770 & 3.18 & 54.80 & 19.33 & 3.03 & 0.0607 & 0.26 & 0.13 & 213 & 290 & 172 & 94 & 76 & 42 & 40 & 715 & 100 & 93 & 36 & 17 & 33 & 33 \\
\hline ASE 92 & 15.67 & 5.88 & 0.736 & 2.43 & 56.62 & 15.95 & 2.18 & 0.0513 & 0.21 & 0.10 & 237 & 179 & 116 & 71 & 63 & 32 & 33 & 837 & 76 & 80 & 31 & 15 & 23 & 22 \\
\hline ASE 93 & 12.85 & 6.22 & 0.767 & 2.55 & 57.48 & 17.01 & 2.39 & 0.0528 & 0.38 & 0.11 & 239 & 213 & 122 & 75 & 64 & 35 & 39 & 846 & 88 & 91 & 33 & 13 & 30 & 24 \\
\hline ASE 94 & 19.90 & 6.50 & 0.711 & 2.13 & 50.52 & 17.33 & 2.27 & 0.0537 & 0.30 & 0.11 & 213 & 214 & 116 & 74 & 71 & 37 & 38 & 799 & 107 & 78 & 30 & 15 & 16 & 21 \\
\hline ASE 95 & 15.73 & 6.02 & 0.730 & 2.80 & 55.08 & 16.69 & 2.37 & 0.0511 & 0.25 & 0.09 & 230 & 218 & 124 & 82 & 64 & 38 & 40 & 748 & 79 & 79 & 32 & 11 & 23 & 25 \\
\hline ASE 96 & 25.08 & 5.94 & 0.657 & 1.58 & 49.13 & 14.90 & 2.12 & 0.0532 & 0.27 & 0.11 & 206 & 231 & 93 & 67 & 64 & 33 & 38 & 742 & 78 & 74 & 30 & 15 & 17 & 23 \\
\hline ASE 97 & 15.10 & 6.24 & 0.748 & 2.64 & 55.40 & 16.91 & 2.39 & 0.0538 & 0.26 & 0.10 & 237 & 189 & 122 & 76 & 66 & 35 & 36 & 720 & 85 & 90 & 32 & 16 & 26 & 24 \\
\hline ASE 98 & 17.87 & 5.45 & 0.631 & 2.73 & 54.22 & 15.50 & 2.95 & 0.0702 & 0.29 & 0.11 & 220 & 266 & 136 & 64 & 56 & 29 & 40 & 710 & 88 & 86 & 32 & 14 & 16 & 18 \\
\hline ASE 99 & 11.84 & 4.99 & 0.724 & 2.30 & 63.40 & 14.17 & 2.08 & 0.0533 & 0.18 & 0.09 & 262 & 179 & 102 & 69 & 56 & 30 & 40 & 650 & 79 & 89 & 32 & 16 & 13 & 18 \\
\hline ASE100 & 12.32 & 5.65 & 0.728 & 2.74 & 58.90 & 16.55 & 2.56 & 0.0543 & 0.21 & 0.13 & 240 & 188 & 129 & 78 & 64 & 37 & 43 & 620 & 88 & 90 & 32 & 19 & 25 & 23 \\
\hline ASE101 & 19.70 & 5.18 & 0.583 & 2.83 & 53.83 & 14.82 & 2.56 & 0.0554 & 0.18 & 0.10 & 177 & 238 & 117 & 65 & 59 & 27 & 36 & 653 & 99 & 74 & 30 & 11 & 28 & 22 \\
\hline ASE102 & 13.61 & 6.54 & 0.776 & 2.67 & 55.37 & 17.95 & 2.47 & 0.0559 & 0.24 & 0.11 & 240 & 197 & 132 & 78 & 69 & 38 & 45 & 917 & 94 & 83 & 31 & 15 & 30 & 21 \\
\hline ASE103 & 14.25 & 6.36 & 0.766 & 2.52 & 56.01 & 17.07 & 2.42 & 0.0549 & 0.28 & 0.10 & 243 & 192 & 124 & 76 & 72 & 38 & 42 & 689 & 88 & 87 & 33 & 16 & 23 & 26 \\
\hline ASE104 & 11.36 & 6.46 & 0.759 & 2.86 & 57.28 & 17.78 & 2.85 & 0.0515 & 0.28 & 0.13 & 230 & 325 & 157 & 88 & 71 & 38 & 47 & 655 & 101 & 98 & 34 & 13 & 33 & 31 \\
\hline ASE105 & 19.80 & 6.41 & 0.689 & 2.55 & 50.88 & 16.81 & 2.26 & 0.0485 & 0.25 & 0.11 & 204 & 248 & 116 & 77 & 67 & 39 & 36 & 846 & 85 & 79 & 30 & 15 & 21 & 25 \\
\hline ASE106 & 22.30 & 5.20 & 0.625 & 2.01 & 54.10 & 13.31 & 1.89 & 0.0478 & 0.25 & 0.10 & 221 & 225 & 86 & 61 & 55 & 30 & 32 & 757 & 89 & 76 & 30 & 13 & 26 & 20 \\
\hline ASE107 & 24.02 & 6.64 & 0.674 & 2.21 & 46.83 & 16.64 & 2.37 & 0.0531 & 0.27 & 0.12 & 184 & 229 & 113 & 75 & 70 & 39 & 36 & 764 & 99 & 77 & 31 & 13 & 20 & 27 \\
\hline ASE108 & 20.62 & 6.16 & 0.672 & 2.45 & 51.19 & 16 & 2.23 & 0.0513 & 0.32 & 0.13 & 201 & 220 & 114 & 73 & 68 & 38 & 36 & 806 & 84 & 81 & 31 & 12 & 22 & 29 \\
\hline ASE109 & 16.71 & 6.85 & 0.768 & 1.55 & 52.32 & 1 & 2.39 & 0.0 & 0.92 & 0.13 & 224 & 218 & 165 & 83 & 76 & 42 & 44 & 839 & 76 & 92 & 32 & 15 & 26 & 24 \\
\hline ASE110 & 21.7 & 6.03 & 0.660 & 2.06 & 50.79 & 15 & 2.25 & 0.0 & 0 & & 191 & 250 & 107 & 74 & 68 & 35 & 39 & 754 & 90 & 75 & 29 & 11 & 18 & 30 \\
\hline ASE111 & 20.47 & 4.81 & 0.604 & 1.5 & 57.75 & 12.4 & 1.76 & 0.0 & 0.3 & 0.10 & 220 & 204 & 92 & 61 & 49 & 30 & 33 & 643 & 83 & 73 & 29 & 14 & 11 & 19 \\
\hline \multicolumn{25}{|c|}{ plaine de Levrau $(n=8)$} \\
\hline ASE 84 & 9.14 & 6.63 & 0 & 2. & e & & 2. & & 0 & 2 & 244 & 328 & 153 & 95 & 69 & 41 & 42 & 985 & 101 & 102 & 35 & 17 & 20 & 34 \\
\hline 85 & 9.89 & 6.66 & 0.80 & 3.00 & 53 & & 2.98 & & 0.2 & 0.12 & 241 & 289 & 162 & 93 & 82 & 40 & 53 & 927 & 87 & 95 & 35 & 11 & 35 & 30 \\
\hline 86 & 6.69 & 6.59 & 0.823 & 3.08 & 59.97 & 18 & 3.27 & & 0.25 & 0.13 & 253 & 286 & 155 & 94 & 74 & 46 & 50 & 1225 & 112 & 102 & 37 & 14 & 41 & 28 \\
\hline ASE 87 & 9.96 & 6.75 & 0.795 & 3.12 & 57.08 & 18.73 & 2.90 & 0.0580 & 0.28 & 0.12 & 236 & 293 & 167 & 94 & 78 & 43 & 46 & 924 & 100 & 101 & 36 & 12 & 35 & 27 \\
\hline ASE 88 & 11.45 & 6.48 & 0.772 & 3.00 & 57.05 & 17.96 & 2.70 & 0.0556 & 0.23 & 0.10 & 238 & 320 & 154 & 87 & 72 & 40 & 42 & 753 & 103 & 94 & 32 & 9 & 35 & 29 \\
\hline ASE 89 & 7.84 & 6.64 & 0.828 & 3.10 & 59.02 & 18.80 & 3.13 & 0.0554 & 0.24 & 0.12 & 261 & 266 & 164 & 90 & 71 & 44 & 52 & 1091 & 92 & 105 & 37 & 13 & 33 & 29 \\
\hline ASE 90 & 12.68 & 6.76 & 0.743 & 2.88 & 55.04 & 18.49 & 2.71 & 0.0604 & 0.29 & 0.13 & 209 & 353 & 162 & 91 & 77 & 42 & 36 & 813 & 114 & 90 & 34 & 14 & 33 & 31 \\
\hline \multicolumn{25}{|l|}{\begin{tabular}{|l} 
Arles \\
\end{tabular}} \\
\hline Nom & $\mathrm{CaO}$ & $\mathrm{Fe}_{2} \mathrm{O}_{3}$ & $\mathrm{TiO}_{2}$ & $\mathrm{~K}_{2} \mathrm{O}$ & $\mathrm{SiO}_{2}$ & $\mathrm{Al}_{2} \mathrm{O}_{3}$ & MgO & $\mathrm{MnO}$ & $\mathrm{Na}_{2} \mathrm{O}$ & $\mathrm{P}_{2} \mathrm{O}_{5}$ & $\mathrm{Zr}$ & $\mathrm{Sr}$ & $\mathrm{Rb}$ & $\mathrm{Zn}$ & $\mathrm{Cr}$ & $\mathrm{Ni}$ & La & $\mathrm{Ba}$ & V & $\mathrm{Ce}$ & $Y$ & Th & $\mathrm{Pb}$ & $\mathrm{Cu}$ \\
\hline \multicolumn{25}{|c|}{ Trinquetaille $(n=7)$} \\
\hline ASE 58 & 9.77 & 5.18 & 0.725 & 2.70 & 63.72 & 14.45 & 1.90 & 0.0 & 1.04 & 0.24 & 221 & 374 & 123 & 74 & 87 & 57 & 35 & 487 & 110 & 78 & 29 & 9 & 31 & 24 \\
\hline ASE 59 & 11.57 & 5.70 & 0.714 & 2.8 & 58 & 99 & 2.37 & 0.0 & 1.10 & 0.18 & 190 & 375 & 137 & 90 & 98 & 64 & 41 & 494 & 110 & 88 & 30 & 15 & 23 & 30 \\
\hline ASE 60 & 10.73 & 5.39 & 0.726 & 2.74 & 62.02 & 14.72 & 2.18 & 0.0939 & 1.08 & 0.16 & 214 & 337 & 135 & 89 & 95 & 59 & 32 & 442 & 109 & 85 & 29 & 10 & 23 & 30 \\
\hline ASE 61 & 14.24 & 5.90 & 0.681 & 2.99 & 55.89 & 16.04 & 2.78 & 0.1040 & 1.03 & 0.17 & 149 & 408 & 149 & 78 & 111 & 65 & 33 & 451 & 132 & 77 & 25 & 9 & 24 & 37 \\
\hline ASE 62 & 10.95 & 5.57 & 0.727 & 2.80 & 60.80 & 15.42 & 2.25 & 0.0958 & 1.09 & 0.13 & 205 & 335 & 137 & 85 & 100 & 61 & 28 & 448 & 108 & 83 & 28 & 15 & 14 & 28 \\
\hline ASE 63 & 14.08 & 5.63 & 0.677 & 2.75 & 57.45 & & 2.61 & 0.0991 & 1.10 & 0.19 & 170 & 399 & 137 & 81 & 101 & 62 & 33 & 456 & 107 & 86 & 29 & 6 & 53 & 33 \\
\hline ASE 64 & 11.75 & 6.04 & 0.729 & 2.97 & 57.84 & 16.39 & 2.71 & 0.1086 & 1.13 & 0.15 & 174 & 396 & 146 & 82 & 110 & 69 & 36 & 429 & 114 & 82 & 24 & 6 & 58 & 34 \\
\hline \multicolumn{25}{|c|}{ 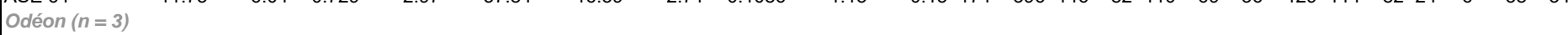 } \\
\hline ASE 65 & 1 & ( & & & & & & & & & 129 & 425 & 171 & 152 & 98 & 65 & 29 & 484 & 129 & 79 & 25 & 4 & 55 & 39 \\
\hline AS & & 6.27 & & 2.64 & & & & & & & & 320 & 128 & 100 & 90 & 62 & 4 & 2 & 103 & 78 & 32 & 10 & 44 & 37 \\
\hline ASE 67 & 18.46 & 5.46 & 0.657 & 3.08 & 54.82 & 14.37 & 1.79 & 0.0965 & 0.85 & 0.23 & 153 & 446 & 121 & 113 & 98 & 49 & 32 & 506 & 125 & 78 & 28 & 9 & 30 & 39 \\
\hline \multicolumn{25}{|c|}{ Nice Cimie } \\
\hline Nom & $\mathrm{CaO}$ & ${ }_{2} \mathrm{U}_{3}$ & $\mathrm{TiO}_{2}$ & $\mathrm{~K}_{2} \mathrm{O}$ & $\mathrm{N}_{2}$ & "2 $2 \sigma_{3}$ & $\mathrm{MgO}$ & $\mathrm{MnO}$ & $\mathrm{NO}_{2} \mathrm{O}$ & $2 \mathrm{C}_{5}$ & $\mathrm{Zr}$ & $\mathrm{Sr}$ & $\mathrm{Rb}$ & $\mathrm{Zn}$ & $\mathrm{Cr}$ & $\mathrm{Ni}$ & $\mathrm{La}$ & B & V & $\mathrm{Ce}$ & $\mathrm{Y}$ & Th & $\mathrm{Pb}$ & $\mathrm{Cu}$ \\
\hline ASE 1 & 12.75 & 5.00 & 0.592 & 3.31 & & 14.94 & 1.66 & & & 0.20 & 264 & 259 & 164 & 83 & 58 & 32 & 40 & 388 & 55 & 87 & 37 & 0 & 250 & 116 \\
\hline ASE 2 & 17.60 & 5.27 & 0.649 & 3.42 & 54.54 & 15.79 & 1.68 & 0.0482 & 0.58 & 0.25 & 223 & 353 & 163 & 89 & 72 & 38 & 36 & 473 & 85 & 88 & 38 & 5 & 68 & 43 \\
\hline ASE 4 & 12.95 & 3.03 & 0.554 & 2.43 & 66.22 & 12.20 & 1.25 & 0.0233 & 0.77 & 0.11 & 226 & 295 & 109 & 68 & 72 & 35 & 28 & 311 & 73 & 81 & 15 & 0 & 3030 & 117 \\
\hline \multicolumn{25}{|c|}{ Hyères, Olbia $(n=3)$} \\
\hline Nom & $\mathrm{CaO}$ & $\mathrm{Fe}_{2} \mathrm{O}_{3}$ & $\mathrm{TiO}_{2}$ & $\mathrm{~K}_{2} \mathrm{O}$ & $\mathrm{SiO}_{2}$ & $\mathrm{Al}_{2} \mathrm{O}_{3}$ & $\mathrm{MgO}$ & $\mathrm{MnO}$ & $\mathrm{Na}_{2} \mathrm{O}$ & ${ }_{2} \mathrm{O}_{5}$ & $\mathrm{Zr}$ & $\mathrm{Sr}$ & $\mathrm{Rb}$ & $\mathrm{Zn}$ & $\mathrm{Cr}$ & $\mathrm{Ni}$ & La & $\mathrm{Ba}$ & V & Ce & $Y$ & Th & $\mathrm{Pb}$ & $\mathrm{Cu}$ \\
\hline ASE 38 & 0.72 & 7.73 & 0.864 & 2.89 & 61.07 & 24.90 & 0.98 & 0.0187 & 0.59 & 0.06 & 259 & 98 & 158 & 58 & 96 & 35 & 51 & 573 & 126 & 106 & 36 & 18 & 58 & 26 \\
\hline ASE 39 & 1.04 & 8.03 & 0.810 & 3.19 & 61.01 & 23.20 & 1.81 & 0.0289 & 0.66 & 0.06 & 190 & 98 & 203 & 105 & 88 & 49 & 48 & 587 & 126 & 111 & 34 & 13 & 52 & 28 \\
\hline ASE 40 & 0.58 & 7.79 & 0.863 & 2.93 & 61.04 & 25.00 & 0.98 & 0.0183 & 0.57 & 0.06 & 253 & 98 & 161 & 59 & 89 & 39 & 49 & 579 & 129 & 107 & 36 & 18 & 44 & 28 \\
\hline
\end{tabular}

